\title{
Manufacturing Planning over Alternative Facilities: Modeling, Analysis and Algorithms
}

\author{
S. David Wu and Hakan Golbasi \\ Manufacturing Logistics Institute, Department of IMSE, Lehigh University, \\ Bethlehem, Pennsylvania 18015
}

\begin{abstract}
We propose a planning model for multiple products manufactured across multiple manufacturing facilities sharing similar production capabilities. The need for crossfacility capacity management is most evident in high-tech industries that have capitalintensive equipment and short technology life cycle. Our model is based on an emerging practice in these industries where product managers from business units dictate manufacturing planning in facilities that are equipped to produce their products. We propose a multicommodity flows network model where each commodity represents a product and the network structure represents linked manufacturing facilities capable of producing the products. We analyze in depth the product-level (single-commodity, multifacility) subproblem. We prove that even the uncapacitated, general-cost version of this subproblem is NP-complete. We develop a shortest-path algorithm for this problem and show that it achieves optimality under special cost structures. We analyze and pinpoint specific cases where the algorithm fails to produce optimal solutions. To solve the overall (multicommodity) planning problem we develop a Lagrangian decomposition scheme, which separates the planning decisions into a number of single-product, multifacility subproblems and a resource subproblem. Through extensive computational testing, we demonstrate that in the context of product-based decomposition the shortest path algorithm is an effective heuristic for the MIP subproblem, yielding high quality solutions with only a fraction (roughly $2 \%$ ) of the computer time.
\end{abstract}

\section{Introduction}

This research is motivated by production problems in the electronics, semiconductor and telecommunication industries. These industries struggle with their production planning problems in an increasingly complex and rapid changing supply chain environment. Specifically, to better utilize their capital-intensive equipment they are pressured to produce a wide variety of products 
in each of their production facilities. However, since these products may each belong to a different supply channel operating under different delivery and outsourcing contracts and demand characteristics, production planning decisions are often relegated to product managers who are most familiar with their specific customer and supplier issues. On the other hand, production managers must consider resource consolidation and capacity management issues in a consistent fashion across manufacturing facilities. These competing viewpoints complicate supply chain planning significantly.

Coordinating production under complex supply structure is not a new problem. However, two recent trends in these industries exacerbate the intensity of the problem. First, the trend toward increased market responsiveness intensifies the inter-dependency within the supply chain. In the past, excess inventory was generally used to reduce the impact of variation across different facilities. Today, most manufacturers are moving away from carrying substantial inventories. Second, the rate of technological innovation significantly shortens the life span of manufacturing equipment, which in term increases the cost of manufacturing capacity. This combined with increased product variety and decreased product volumes prompt manufactures to cross-load their manufacturing facilities.

In this paper, we focus on cross-facility operational planning faced by high-tech manufacturing companies in a supply chain environment. Our research is motivated by experiences in production management system at a major semiconductor manufacturer for their world-wide supply base, and by the quantitative supply chain literature. Quantitative analysis of supply chain management has been focusing on channel design in general and stocking policies in specific using extensions of inventory, game-theoretic, and strategic models (Tayur et al., 1999). Cohen and Lee (1988)(1989), Sterman (1989) and Davis (1993) are among the pioneers who made significant early contributions. Various development of these models remain an area of active research (c.f., Tayur et al., 1999, Lee, et al. 1995, Hahm and Yano 1995a,b and Arntzen, et al, 1995). In addition to supply chain design, coordinating various aspects of supply chain operations has been an area of active research as well. This line of work is exemplified by Vidal and Goetschalckx (1997), Hahm and Yano (1995a,b), and Ertogral and Wu (1999). A related, but distinctively different, line of research focuses on the extension of production models in the context of MRP systems (c.f. Billington, et al., 1983, Carlson and Yano, 1983, Gupta and Brennan 1995). The focus here is manufacturing planning in the context of multilayer and multi-facility production. This line of research is rooted from multi-level, multiperiod, capacitated lot-sizing models. A number of surveys (c.f., Bahl et al. 1987, Goyal and Gunasekaran, 1990; Baker 1993; Kimms 1997) provide an excellent overview for research in this 
area. While our proposed model can be linked directly to the multi-level lot sizing literature, it has two distinctive features that are not previously addressed: first is the explicit consideration of facility selection decisions. Most existing work assumes either a single facility, or multiple tiers of facilities as defined by the product structure, but the facility selection decisions are given $a$ priori. Second, we study a single-item subproblem (with facility selection decisions) that has been overlooked in the literature. Unlike it's single-facility counterpart, this subproblem is NPcomplete even in the uncapacitated case. Despite of this, we show analytically and empirically that a shortest path algorithm could be extremely effective.

\section{A Multi-Facility Production Model}

We now consider a multi-facility production model where a set of end-items is to be produced in multiple facilities over multiple periods. Each end-item has a bill of material described by a product structure. In addition, there is a supply structure where a set of alternative facilities could be setup to produce each item described in the product structure. Figure 1 illustrates the product and the supply structure. The product structure in Figure 1 can be represented by a "gozinto" structure common in the lot-sizing literature. The gozinto structure is specified in an $n \times n$ matrix $\left[a_{i k}\right]$ where $a_{i k}$ is the number of item $i$ that is (directly) needed to produce one unit of item $k$. In addition to the gozinto structure we define a supply structure matrix $\left[r_{i j}\right]$ where $r_{i j}=1$ if facility $j$ could be used to produce item $i$, and $r_{i j}=0$ otherwise.

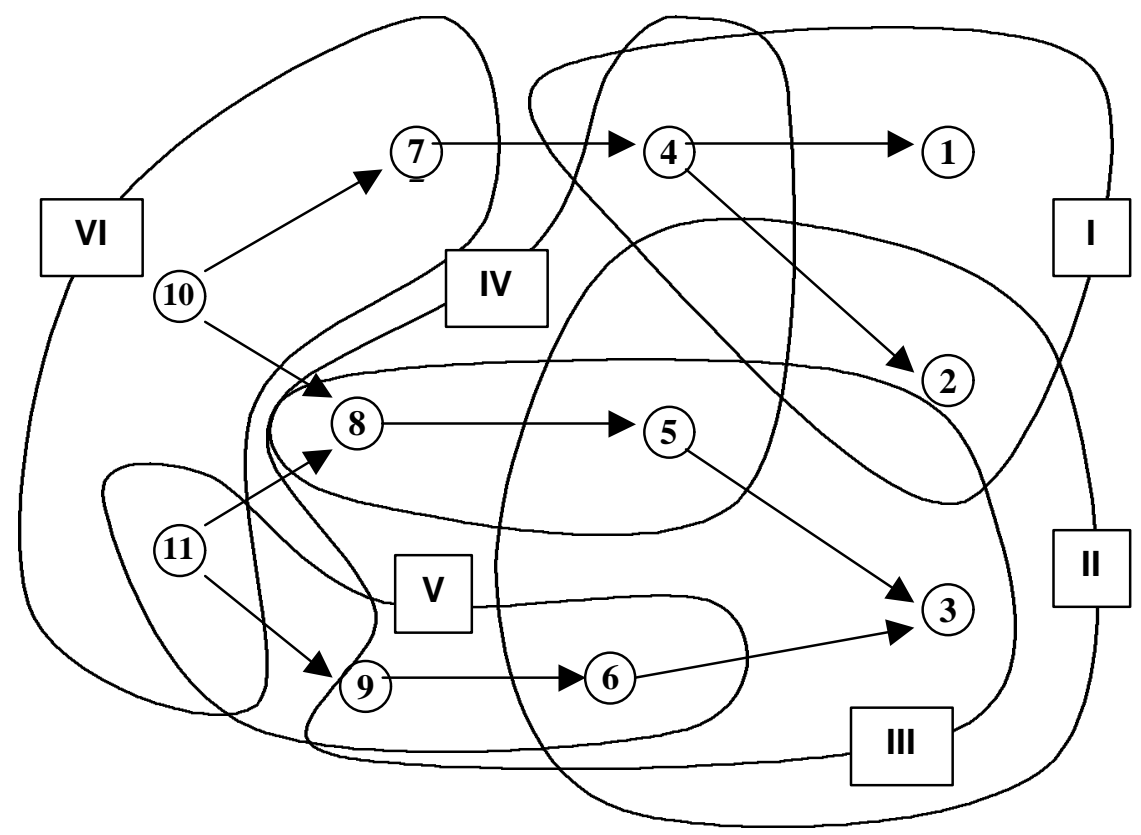

Figure 1. A Supply Network with Three End-Items (1,2 and 3), Six Facilities (I to VI) and a Maximum of Three Alternative Facilities for an Item 


\section{A Multicommodity Flows Model}

The above multi-facility production problem is complex in that the facility selection decisions are combined with multi-stage, multi-item, multi-period production decisions. To approach this problem we take the viewpoint of a subset of manufacturing facilities in the supply network. Each manufacturing facility can produce a variety of products (items) $(i=1,2, \ldots, \mathrm{n})$ over multiple periods $(t=1,2, . ., \mathrm{T})$, while each item $i$ can be produced in a specified set of alternative facilities $\left(j=1,2, \ldots, \mathbf{J}_{i}\right)$. Each facility can be setup to perform a certain production processes with a setup cost. Now consider a multicommodity network $\mathrm{G}(\mathrm{N}, \mathrm{A})$ where each item $i$ corresponds to a commodity in the network. Let $D_{t}{ }^{i}$ denote internal demands for item $i$ in period $t$ as defined by the end-item demand and the product structure. Suppose $D_{t}{ }^{i}$ can be generated a priori using standard MRP explosion, we can then define a multicommodity flows network corresponding to the supply network as shown in Figure 2. This multicommodity flows network has three main parts: a set of source nodes representing demand dispatching points, a set of sink nodes representing demand fulfillment points, and a set of production subnets in between each represents multi-period production to be carried out by a facility. Each commodity (product) $i$ has a source node $s^{i}$, and T sink nodes $d^{i}{ }_{t}$, one for each period t.

We first describe the overall network structure. The input flow for source node $\mathrm{s}^{i}$ is the total demand over $\mathrm{T}$ periods for item $i\left(\sum_{t=1}^{4} D_{t}^{i}\right)$, and the outflow on sink note $d_{t}^{i}$ as the demands for item $i$ in period $t,\left(D_{t}^{i}\right)$. The presence of an arc going from source nodes $\mathrm{s}^{i}$ to facility subnet $j$ signifies the fact that facility $j$ can be setup to produce item $i$. These arcs are specified by the supply structure matrix, i.e., there is an arc $(i, j)$ corresponding to each non-zero entry of matrix $\left[r_{i j}\right]$. The subnets between the set of source and sink nodes represent production facilities shared by the products. The arcs going from facility subnets $j=1,2, . ., \mathrm{J}_{i}$, period $t$, to sink node $d^{i}{ }_{t}$ represents that item $i$ 's demand in period $t$ is to be fulfilled by the production and/or inventory from all (or some) of its $J_{i}$ alternative facilities. Note that the production subnet can be further "customized" according the structure of each manufacturing facility. For example, the structure in Figure 2 shows the familiar single-stage multi-period lot-sizing model. This can be extended to a multi-stage model (c.f., Afentakis 1984) also shown in the figure, or other multi-period models. To streamline the analysis, we assume single-stage facility subnets throughout the paper.

We now characterize arc labels in the multicommodity network. Each arc in the network is characterized by $\left(f^{i}, c^{i}, u\right)$ : arc flow $f^{i}$, per unit cost $c^{i}$ and arc capacity $u$. The interpretation of these values varies according to the types of arcs. The arcs going from source nodes $s^{i}$ to the 
facility subnets $j$ 's are facility selection $\operatorname{arcs} A_{s} \subset A$, characterized by $\left(x_{j}^{i}, c_{j}^{i}, u_{i j}\right): x_{j}^{i}$ represents the total production to be performed on facility $j$ over $t=1, \ldots, T, c_{j}^{i}$ represents the arc cost that quantifies the differences among facilities (e.g., quality history, reputation), and capacity $u_{i j}$ represents the maximum amount of item $i$ that can be produced in facility $j$. When the dynamic lot-sizing model is used in the production subnet, two types of arcs are used: arcs going from left to right are production arcs $A_{p} \subset A$, characterized by $\left(x_{j t}^{i}, c_{j t}^{i}, \operatorname{cap}_{j t}\right)$ : production quantity $x_{j t}^{i}$, unit production cost $c_{j t}^{i}$, and production capacity $c a p_{j t}=u$. Arcs going from top down are inventory $\operatorname{arcs} A_{I} \subset A$ characterized by $\left(I_{j t}^{i}, h_{j t}^{i}, i n v_{j t}\right)$ : inventory carried from period t to $\mathrm{t}+1, I_{j t}^{i}$, unit inventory holding $\operatorname{cost} c_{j t}^{i}$, and inventory limit $i n v_{j t}=u$. Finally, an arc going from facility $j$ period $t$ to sink node $d^{i}{ }_{t}$ belongs to the demand arcs $A_{d} \subset A$, characterized by $\left(b_{j t}^{i}, r_{j t}^{i}, \operatorname{cap}_{j t}\right): b_{j t}^{i}$ represents facility $j$ 's contribution to demand $D_{t}^{i}, r_{j t}^{i}$ represents the transportation cost from facility $j$ to the demand point $i$, and $\operatorname{cap}_{j t}$ is the transportation capacity in period $t$.
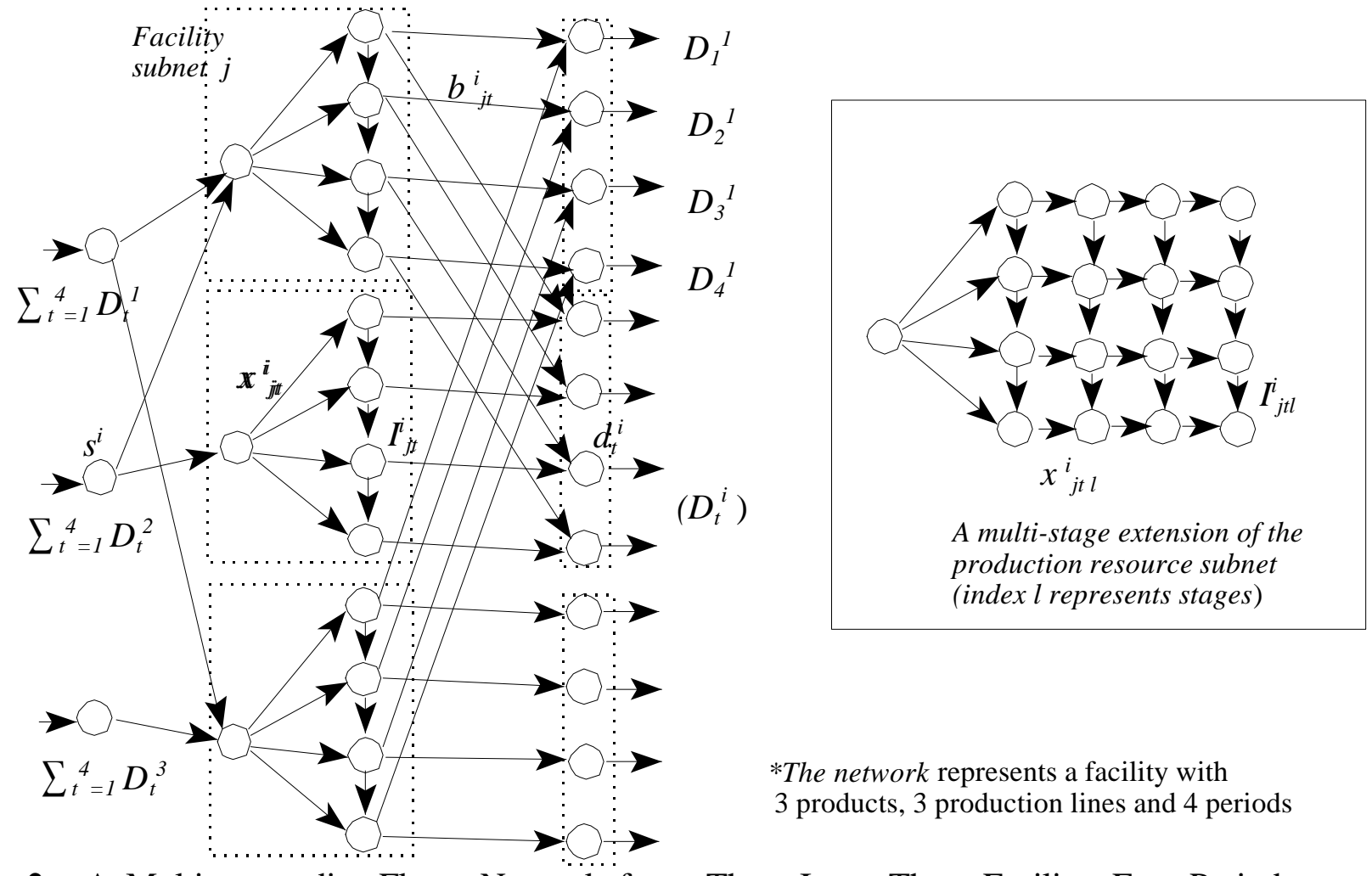

Figure 2. A Multicommodity Flows Network for a Three-Item, Three-Facility, Four-Period Model

Given the above specification, we can define the general multicommondity constrains (1.1) and (1.2) as follows:

General arc capacity constraints for all arcs 


$$
\begin{array}{ll}
x_{j}^{i} \leq u_{i j} & \forall(i, j) \in A_{s} \\
\sum_{i=1}^{n} \beta_{j t}^{i} f_{j t}^{i} \leq u_{j t} & \forall(j, t) \in A_{p} \cup A_{I} \cup A_{d}
\end{array}
$$

where $\beta$ is the capacity consumption rate. Denote $a$ the node-arc incidence matrix for the multicommodity flows network $\mathrm{G}(\mathrm{N}, \mathrm{A})$ and $e^{i}$ the net balance flows for commodity $i$. The mass balance constraints are as follows:

\section{Mass balance constraints for each commodity}

$$
\text { a } f^{i}=e^{i} \quad \forall i \in N
$$

In addition to the general multicommodity flows constraints as specified by the network structure G(N,A), we define additional constraints for each facility submodel. Consider the multi-period lot-sizing model we use for all facility subnets. While the inventory balance constraints are part of the mass balance constraints (1.2), we need to define additional constraints due to setup. Let

$\alpha_{j t}^{i}$ denote the rate of capacity consumption for setup activities, $\delta_{j t}^{i}$ a binary variable indicating the existence of a setup for item $i$ at facility $j$ at period $t$. The production specific constraints are as follows:

Production capacity constraints for production and setup

$$
\sum_{i=1}^{n} \sum_{j=1}^{J_{i}}\left(\beta_{j t}^{i} x_{j t}^{i}+\alpha_{j t}^{i} \delta_{j t}^{i}\right) \leq c a p_{j t} \quad \forall(j, t) \in A_{p}
$$

Setup constraints

$$
x_{j t}^{i} \leq M q_{j t}^{i} \quad \forall i \in N,(j, t) \in A_{p}
$$

Up to this point we ignore the fact that the items entering the production facilities (as represented by the multicommodity flows network $\mathrm{G}(\mathrm{N}, \mathrm{A})$ ) has an underlying product structure $\left[a_{i k}\right]$. To model the supply structure as well as the product structure across multiple tiers of a supply chain, we will need to include additional constraints which specify the relationship among demands of items over time $D_{t}^{i}, i=1, \ldots, n, t=1, \ldots, T$. First of all, the demand for end-items, $N_{0} \subseteq$ $N$, must be satisfy. Although this is already implied in the mass balance constraints (1.2), we will restate it for clarity.

$$
\begin{aligned}
& \text { Demand for end item must be satisfied } \\
& \qquad \sum_{j=1}^{m} b_{j t}^{i}=D_{t}^{i} \quad \forall t, i \in N_{0}
\end{aligned}
$$

The end-item demand triggers the internal demands in the supply chain as defined by the product structure. Denote $L_{k}$ the lead-time for item $k$, we can define the following relationship: 
Demand internal the supply chain must be satisfied in each period

$$
\sum_{j=1}^{m} b_{j t}^{i}=\sum_{k=1}^{n} a_{i k} D_{t+L_{k}}^{k} \quad \forall t, i \in N-N_{0}
$$

Denote $c_{j t}^{i}$ and $h_{j t}^{i}$, respectively, the production and the inventory holding costs for item $i$ at facility $j$ during period $t$, and $K_{j}^{i}$, the set-up cost for item $i$ at facility $j$, and $r_{j t}^{i}$ the unit transportation cost from facility $j$ to the demand point. We state an objective function with these cost components defined for the production subnet. A cost component unique to each product $i$, say $S_{i}\left(f^{i}, c^{i}, u\right)$, could be added to the objective to reflect special requirements imposed by the supply channel of $i$. Since this does not affect our analytic results significantly, we will not consider this cost terms here. Thus, a multicommodity flows formulation of the multi-facility production problem $(\mathbf{P})$ is as follows:

\section{Problem (P):}

$$
\text { Minimize } z=\sum_{t=1}^{T} \sum_{i=1}^{n} \sum_{j=1}^{J_{i}}\left(c_{j t}^{i} x_{j t}^{i}+K_{j}^{i} \delta_{j t}^{i}+h_{j t}^{i} I_{j t}^{i}+r_{j t}^{i} b_{j t}^{i}\right)
$$

s.t.

$\langle$ General Multicommodity Flows Constraints (1.1)-(1.2)〉

$\langle$ Production Specific constraints (2.1)-(2.2)>

$<$ Supply-Chain Specific constrains (3.1)-(3.2)>

(4) nonnegativity constraints

$$
f^{i}, x_{j t}^{i}, \delta_{j t}^{i}, I_{j t}^{i}, b_{j t}^{i} \geq 0
$$

(5) binary constraints

$$
\delta_{j t}^{i} \in(0,1)
$$

It is useful to note that in this multicommodity flows model, only the capacity constraints (1.1) and (2.1) are bundling constraints. All the other constraints can be decomposed by commodity (product). (P) is a multi-period, multi-item, multi-facility production planning model.

\section{Model Analysis}

Note that since the mass balance constraints (1.2) imply $\sum_{j=1}^{m} b_{j t}^{i}=D_{t}^{i}, \forall i \in N$, constraints (3.2) in effect specify the relationship between the demands of item $i$ and the items ( $k$ 's) which use $i$ as a component, i.e.,

$$
D_{t}^{i}=\sum_{k=1}^{n} a_{i k} D_{t+L_{k}}^{k}, \forall t, i \in N-N_{0}
$$


Consider a set of facilities in a manufacturing supply chain as depicted in Figures 2. Suppose the end-item demand is stationary, the relationship in (3.3) suggest that it is possible to generate $D_{t}^{i}$ a priori using a BOM explosion mechanism frequently used in MRP systems, i.e., we may treat $D^{i}$ as parameters generated and fixed a priori for the multicommodity flows model such that (3.2) is always satisfied. This simplifies the facility selection decisions considerably since we do not need to make the multi-tier production planning decisions all at the same time. This corresponds to the practice in most industries where the demand information across different tiers of the supply chain are announced ahead of time according to built-in leadtimes (rather than pulled instantaneously).

To explore special subproblem structures that will later help the solution of model $(\mathbf{P})$, we consider two submodels in the following sections.

\subsection{Uncapacitated Single-Item, Multi-Facility Model without Transportation Costs}

As stated above, model $(\boldsymbol{P})$ can be decomposed by commodity after relaxing the bundling capacity constraints (1.1) and (2.1). We first consider a capacity-relaxed single-item, multiplefacility subproblem (without transportation costs) for commodity $i$ as follows:

(P) Minimize $\sum_{i=1}^{T} \sum_{j=1}^{J_{i}}\left(c_{j t} x_{j t}+K_{j} \delta_{j}+h_{j t} I_{j t}\right)$

s.t. $\quad\langle$ mass balance constraints for commodity $i(1.2)>$

$<$ setup constraint for commodity $i(2.2)>$

$<$ constraints (4)(5) for commodity $i>$

Subproblem $\left(\boldsymbol{P}_{\boldsymbol{i}}\right)$ represents a subset of the decision problem for the product manager of $i$ who must decide where to produce her product among a certified ${ }^{1}$ set of manufacturing facilities $J_{i}$. Note that we dropped the cost component $S_{i}\left(f^{i}, c^{i}, u\right)$ from the objective, hence the facility selection and the transportation costs are assumed to be zero. Figure 3 depicts a subnetwork defined by commodity 1 (for problem $\left(\boldsymbol{P}_{\boldsymbol{1}}\right)$ ) corresponding to the example in Figure 2.

While the above facility-selection/lot-sizing problem has not been well studied, the single-facility, single-item, uncapacitated lot-sizing problem has been studied intensively in the literature. Despite of the binary variables it is well known that this problem can be solved in polynomial time using Wagner-Within type algorithms. In recent years, more efficient implementation of Wagner-Within algorithms has been developed (c.f., Federgruen and Tzur (1991) and Wagelmans et al. (1992)), which has order $O(n \log n)$ or better. Embedding these

\footnotetext{
${ }^{1}$ The notion of facility certification is important in Semiconductor Manufacturing where a product can only produce in a facility that has been pre-certified for quality and yield.
} 
polynomial-time solvable problem as submodels, the multi-item, capacitated lot-sizing problems are frequently solvable in a reasonable amount of time for realistic size problems (c.f. Tempelmeir and Derstroff, 1996). Since a primary new consideration in our model $(\boldsymbol{P})$ is the selection of alternative facilities, it is important to know the structure and the complexity of subproblem $\left(\boldsymbol{P}_{\boldsymbol{i}}\right)$. This analysis is important to the solution of $(\boldsymbol{P})$ since (1) $\left(\boldsymbol{P}_{\boldsymbol{i}}\right)$ has the form of a mixed integer program, and (2) there is no straightforward (efficient) decomposition from the multi-facility case to single-facility. In the following, we first show that $\left(\boldsymbol{P}_{\boldsymbol{i}}\right)$ is $N P$-complete when the holding cost $h_{j t}$ is general and not restricted in sign. We then show that an efficient algorithm exists under a special set of conditions. Later in Section 4 we show computationally that under general cost conditions this algorithm is an effective heuristic, solving most instances of $\left(\boldsymbol{P}_{\boldsymbol{i}}\right)$ optimally.

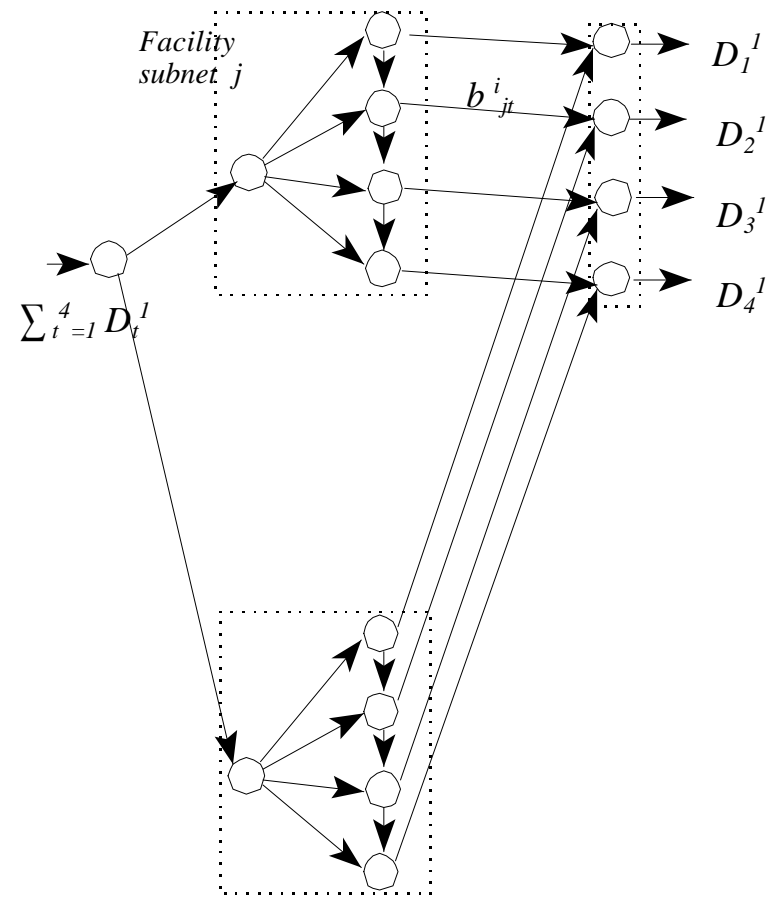

Figure 3. Subnetwork Corresponding to Commodity 1, with 2 alternative facilities, 4 periods

Theorem 1. (Non-splitting property): There exists an optimal solution to the uncapacitated, single-item, multiple facility problem $\left(P_{i}\right)$ such that item i's demand in period $t$ is satisfied by the production or the inventory of exactly one of the $J_{i}$ facilities, i.e., exactly one $b_{j t}^{i}$ among $j=1, . ., J_{i}$ is non-zero $\left(=D_{t}^{i}\right)$ for each period $t$.

Proof: see Appendix. 
As we shall see in the following exploration, the insight provided by the non-splitting property plays an important role in the development of solution algorithms for the uncapacitated subproblem $\left(P_{i}\right)$ as well as the overall problem $(P)$. Nonetheless, despite of its promising outlook, subproblem $\left(P_{i}\right)$ can be only solved efficiently under more restrictive conditions due to the existence of several pathological cases. In the following, we first show that under generalized cost condition subproblem $\left(P_{i}\right)$ is NP-Complete.

Theorem 2. The uncapacitated, single-item, multiple-facility problem is NP-Complete when the inventory holding cost $h_{j t}$ is a generalized cost coefficient not restricted in sign.

Proof: see Appendix.

Upon examining the proof it should be clear why NP-Completeness can be only constructed for the case where arc cost is not restricted in sign. In the following, we show that the NP-complete status remains when the demands or the setup costs are constant.

Corollary 1. The problem stated in Theorem 1 remains NP-Complete when the period demands $D_{t}$ are constant over periods $t=1, \ldots, T$.

Corollary 2. The problem stated in Theorem 1 remains NP-Complete when the setup costs $K_{j}$ are constant across facilities $j=1, \ldots, m$.

In the following, we go on to show that despite of the $N P$-complete status of problem $\left(P_{i}\right)$ and its variations, under mild assumptions a shortest path algorithm solves problem $\left(P_{i}\right)$.

The uncapacitated, single-item, multiple-facility problem $\left(P_{i}\right)$ can be solved in polynomial time using a shortest path algorithm if and only if the following conditions hold:

(i) No simultaneous production of item i over more than one facility can take place in a given period. In other words, $x_{j t}^{i} x_{k t}^{i}=0, \forall i, j, k \neq j, t$.

(ii) No production of item $i$ will be scheduled at all if there is inventory carried over from a previous period in one of the facilities. In other words, $x_{j t}^{i} I_{k t-1}^{i}=0, \forall i, j, k, t$.

Proof: We will state the proof using a familiar graphical representation as in Figure 4. The first row of nodes denotes facility 1 and the ith row denote facility $J_{i}$. There are $T+1$ time epochs:0,1,2,3, and a period is the interval between epochs, i.e., between epochs 0 and 1 is period $t=1$, and between 1 and 2 is period $t=2$, etc. A horizontal arc denotes the production that satisfies all the periods' demand within the time epochs. The arc cost includes production, inventory and setup costs. A vertical arc denotes a switch from one facility to another and the 
cost associated to these arcs are 0. There is an artificial source and sink, arcs adjacent to these nodes have 0 cost. In a general graph for each time epoch there are arcs for all the facility periods. So production of an item may switch from one facility to any other in different periods. From condition (ii), there will be production scheduled for item $i$ only if there is no inventory carried over from a previous period in one of the facilities. In other words, in an optimal solution exactly one arc will be chosen to enter a given node in the network. On the other hand, condition (i) states that there can be no simultaneous production of items $i$ in more than one facility in any given period. In other words, in an optimal solution exactly one arc will be chosen to leave a node in the network. This means that an optimal production schedule corresponds to a source-tosink path in the network, and it corresponds to a shortest cost path.

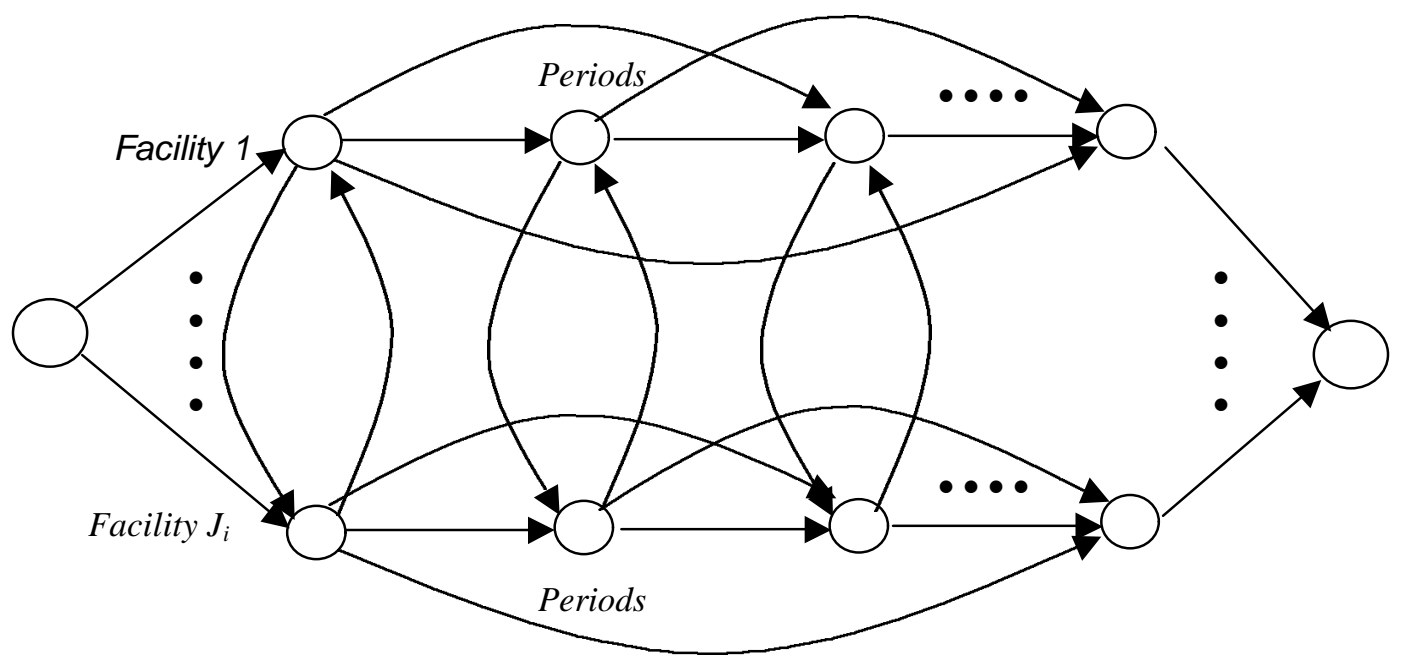

Figure 4. The Multi-Facility Production Problem as a Shortest Path Problem

Unfortunately, there are cases where conditions (i) and (ii) in Proposition 1 do not hold in the optimal solution. This is caused by pathological cases such as the follows: under completely general production and inventory holding costs, it could be optimal to produce $x_{j t}^{i}$ in a facility $j$ in period $t$, holding this amount in the inventory for a future period $t+l(l>1)$ without using it in periods $t+1, \ldots, t+l-1$. Since more than one facility may produce, the demands in periods $t+1, \ldots$, $t+l-1$ could be satisfied by a number of facilities other than $j$. This creates the possibility of "multiple-path" production (see Figure 5). For instance, in Figure 5-(b), a production is started in period 1 at both facility 1 and 2, while the former is used to satisfy demands in periods 2 and 3 , the latter is produced for period 1. This is possible when the production cost for facility 1 in period 1 is higher than that of the facility 2, but the combination of production and holding costs for periods 2 and 3 is lower in facility 1 than that of facility 2 . Note that this particular case violates condition (i) and no shortest path algorithm will identify this as a solution. Similarly, in 


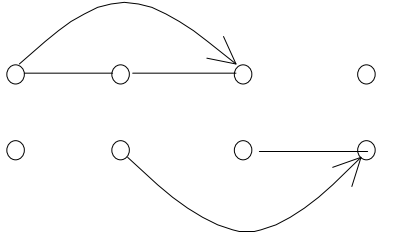

(a)

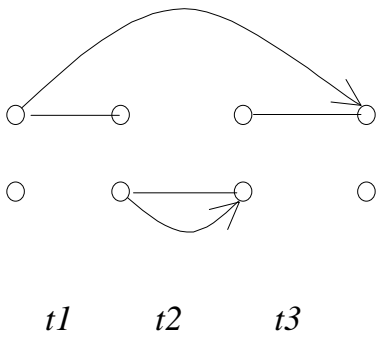

(c)
Facility 1

Facility 2

Facility 1

Facility 2
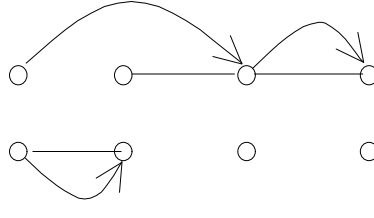

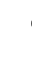

(b)

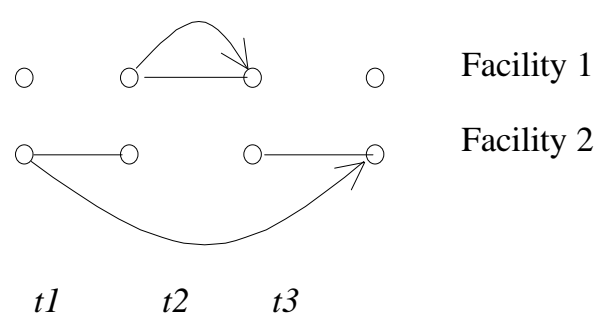

(d)
Facility 1

Facility 2 
(ii) $\quad x_{j t}^{i} I_{k t-1}^{i}=0, \forall i, j, k, t$.

In Case 1 above, it is clear that condition (i) is violated while in Case 2, condition (ii) is violated

The fact that the shortest path algorithm is not always optimal for subproblem $\left(P_{i}\right)$ should not come as a surprise as Theorem 2 shows that the generalized cost version is $N P$ Complete. Nevertheless, the shortest path algorithm is a valid heuristic for subproblem $\left(P_{i}\right)$. In the computational experiments, we will show that the shortest path algorithm is an effective heuristic, yields optimal or near-optimal solutions in most test cases.

\subsection{Uncapacitated Single-Item, Multi-Facility Model with Transportation Costs}

Recall that in the multicommodity flows network (Figure 2) and the single-item subnetwork (Figure 3) a demand $\operatorname{arc} A_{d} \subset A$ goes from facility $j$ period $t$ to demand point $d{ }^{i}{ }_{t}$ is characterized by $\left(b_{j t}^{i}, r_{j t}^{i}, \operatorname{cap}_{j t}\right)$ where $b_{j t}^{i}$ represents facility $j$ 's contribution to demand $D_{t}^{i}, r_{j t}^{i}$ represents the transportation cost from facility $j$ to the demand point $i$, and cap $_{j t}$ is the transportation capacity in period $t$. In Section 3.1 we assume the transportation cost $r_{j t}^{i}=0$ and $\operatorname{cap}_{j t}=\infty$. We will now consider the single-item subproblem where the transportation cost is positive but we will not consider transportation capacity. The subproblem can be stated as follows

$\left(\boldsymbol{P}_{i}\right) \quad$ Minimize $\sum_{t=1}^{T} \sum_{j=1}^{J_{i}}\left(c_{j t} x_{j t}+K_{j} \delta_{j}+h_{j t} I_{j t}+r_{j t} b_{j t}\right)$

s.t. $\quad\langle$ mass balance constraints for commodity $i(1.2)>$

$<$ setup constraint for commodity $i(2.2)>$

$<$ constraints (4)(5) for commodity $i>$

Theorem 3. The uncapacitated, single-item, multiple-facility problem with non-negative transportation cost $r_{j t}$ is NP-Complete.

Proof. See Appendix.

\section{Algorithm Complexity}

We now explore possible algorithms for problem $\left(P_{i}^{\prime}\right)$ and their complexity as related to the number of facilities and number of periods. A first observation is by viewing the single-item, multi-facility problem depicted in Figure 3 as a Directed Steiner Tree Problem (Leibling, 1999): the subnetwork represents the problem graph, while the source, the root of each production subnet, and the demand nodes (on the right) are "compulsory" nodes, the remaining are 
"optional" or Steiner points. The Directed Steiner Tree Problem is to find an arborescence of minimal total length that spans all compulsory nodes using one or more Steiner points. Suppose arc lengths can be determined a priori using production, inventory, and transportation costs, an optimal Steiner solution corresponds to an optimal solution to $\left(P_{i}^{\prime}\right)$. It is known that a dynamic programming algorithm (Dreyfus and Wagner, 1972) solves the Steiner Problem in $O\left[\left(N_{c}+N_{s}\right) 3^{N_{s}}+\left(N_{c}+N_{s}\right)^{2} \cdot 2^{N_{s}}\right]$ where $N_{s}$ is the number of Steiner points (i.e., number of facilities $\times$ number of periods) and $N_{c}$ is the number of compulsory points (i.e., number of periods). While this observation suggests that a dynamic programming algorithm constructed in this fashion is exponential in the number of facilities and period, using the well-known WagnerWithin results, we could improve the complexity significantly. This is stated as follows.

Theorem 4. There exists an algorithm for the uncapacitated, single-item, multiple-facility problem with non-negative transportation cost (Problem $\left.\left(P^{\prime}{ }_{i}\right)\right)$ that is polynomial in the number facilities and exponential in the number of periods.

Proof. Consider a simple algorithm as follows for problem $\left(P^{\prime}{ }_{i}\right)$ :

(1) Assign demands $D_{1}, \ldots, D_{T}$ to facilities $j=1, \ldots, m$ (this takes $O\left(m^{T}\right)$ )

(2) Given the assignment, solve for each facility $j=1, \ldots, m$ an uncapacitated, single-item, singlefacility lot-sizing problem using the Wagner-Within Algorithm (this takes $m \cdot O(T \log T)$ )

The algorithm has complexity $O\left(m^{T+1} \cdot T \log T\right)$, which is polynomial in the number of facilities but exponential in the number of periods.

\section{Special Cost Structure}

Despite of the negative results, the shortest path algorithm remains a viable option under certain cost structures. Specifically, when transportation and production costs are fixed, when there is no incentive to hold inventory for more than four periods without starting a new setup, and when holding inventory in a facility $j$ for two periods always costs more than holding inventory elsewhere for one period (i.e., when holding costs are not dramatically different), all pathological cases stated in Proposition 2 and Figure 5 disappear.

Proposition 3. The uncapacitated, single-item, multiple-facility problem with non-negative transportation cost $\left(P_{i}^{\prime}\right)$ can be solved in polynomial time using a shortest path algorithm if the following conditions hold:

(i) The transportation and the production costs are fixed across facilities and periods

(ii) There is no setup that would last more than four periods, i.e., $\sum_{\tau=t}^{t+3} h_{j \tau} D_{\tau} \geq K_{j}, \forall j$

(iii) Inventory holding costs of any two facilities $j$ and $k$ satisfies the relationship 
$h_{j t}+h_{j, t+1} \geq h_{k, t+1} \quad \forall j, k, t$

It should be intuitive when conditions (i)-(iii) above hold in the data set, the condition in Proposition 2 is eliminated, while conditions $(i)$ and (ii) in Proposition 1 will be satisfied, i.e., the shortest path algorithm will produce optimal solution. These conditions can be thus used as a test to be performed on the data set before calculation starts. It is quite likely that even when the above conditions are violated, the shortest path algorithm remains an effective heuristics. To put this point under solid empirical testing, we conducted two sets of computational experiments: the first experiment simply compares the optimal solutions for $\left(P_{i}\right)$ obtained by a MIP (Mixed Integer Programming) solver vs. solution provided by a shortest path algorithm. The results are summarized in Section 3.4. The second set of experiments is more complicated, here we test the quality of the single-item shortest path algorithm as a subproblem heuristic for the multi-item problem (P). This will be detailed in Section 4.

\subsection{Performance of the Shortest Path Algorithm}

To instantiate the insights gained from the analytic results, we conduct intensive empirical testing by varying setup, holding, production, and transportation costs, the number of facilities and periods combinations, and levels of demand lumpiness. This results in 17,500 instances. We first generate a nominal case as follows:

Setup cost , $K_{j} \sim$ Uniform [1500,3500]

Production cost, $c_{j t} \sim$ Uniform $[5,15]$

Transportation cost, $r_{j t} \sim$ Uniform [5,15]

Holding cost, $h_{j t} \sim$ Uniform $[5,15]$

Number of facilities: 4

Number of periods: 6

Demand $D_{t} \sim$ Uniform $[5,15]$

No demand lumpiness

Demand lumpiness is used to generate clustered demand patterns more closely resemble practical situations. While generating random demand for $D_{t}$ period $t$, we first generate a random number between 0 and 1 , if it is less than the current lumpiness threshold $\zeta$, then $D_{t}$ is set to 0 , otherwise, $D_{t}$ set to the generated demand. We adjust the demand levels such that the expected demand remains the same as the base case.

By varying one or more factors from the nominal case, we generate three groups of test problems as follows: 
(Group One): Fixed setup cost across all periods, i.e., setup cost is generated once and fixed for all periods (by varying the following cost factors, we generate 65 sub-groups where 100 replications are generated for each subgroup) al-a10: varying the setup cost levels from Uniform [0,500] to Uniform [5000,10000] a11-a20: varying the holding cost levels from Uniform [1,5] to Uniform [100,200] a21-a30: varying the production cost levels from Uniform [1,5] to Uniform [100,200] a31-a40: varying the transportation cost levels from Uniform [1,5] to Uniform[100,200] a41-a60: varying the number of facilities $\left(J_{i}\right) /$ number of periods $(T)$ combinations, or $\left|J_{i}\right| \times|T|$ where $\left|J_{i}\right|=2,3,4,5,6$ and $|T|=6,8,10,12$ a61-a65: varying the demand lumpiness threshold $\zeta=0,0.2,0.3,0.4,0.5$

(Group Two): Variable setup cost across periods, i.e., setup costs are randomly generated for each period $t$. Again, 65 sub-groups are generated as above with 100 replications each.

(Group Three): Fixed production and transportation costs, i.e., the production and transportation costs are fixed to their expected value (10). This eliminates subgroups a21-a40 above, resulting in 45 subgroups with 100 replications each.

A summary of the test results is given in Figure 6. As shown in the Figure, except for subgroups $a 37-a 40$, the shortest path algorithm produces nearly identical solutions as the MIP (i.e., less then 3 occurrences per 100 instances, with a maximum solution deviation less than $0.2 \%$ ). When more significant deviations are observed in subgroups a37-a40 (cases where the transportation costs are high at Uniform [40,80], .., Uniform [100,200], respectively), although the number of occurrences are much more pronounces (up to 49 per 100 instances), the maximum amount of deviation is still under $0.5 \%$ in all cases. The number of occurances are more pronounced when the setup costs are variable across periods (Group Two). Now consider the Group Three results: there is no deviation between shortest path and MIP solutions in any of the 4,500 cases tested. Consider this result along with Proposition 3. It is interesting to note that when the production and transportation costs are fixed (condition (i) in the proposition), even when conditions (ii) and (iii) do not hold, the shortest path algorithm produces optimal solutions. These results confirm our insight from Proposition 2 that pathological cases exist where the shortest path algorithm produces sub-optimal solutions; upon examining these cases, we found that they are indeed caused by circumstances depicted in Figure 5. On the other hand, the empirical results show that the shortest path algorithm is a very effective heuristic for the single-item, multi-facility subproblem. In the following section, we consider this singleitem subproblem in the context of the Multi-Item problem. 

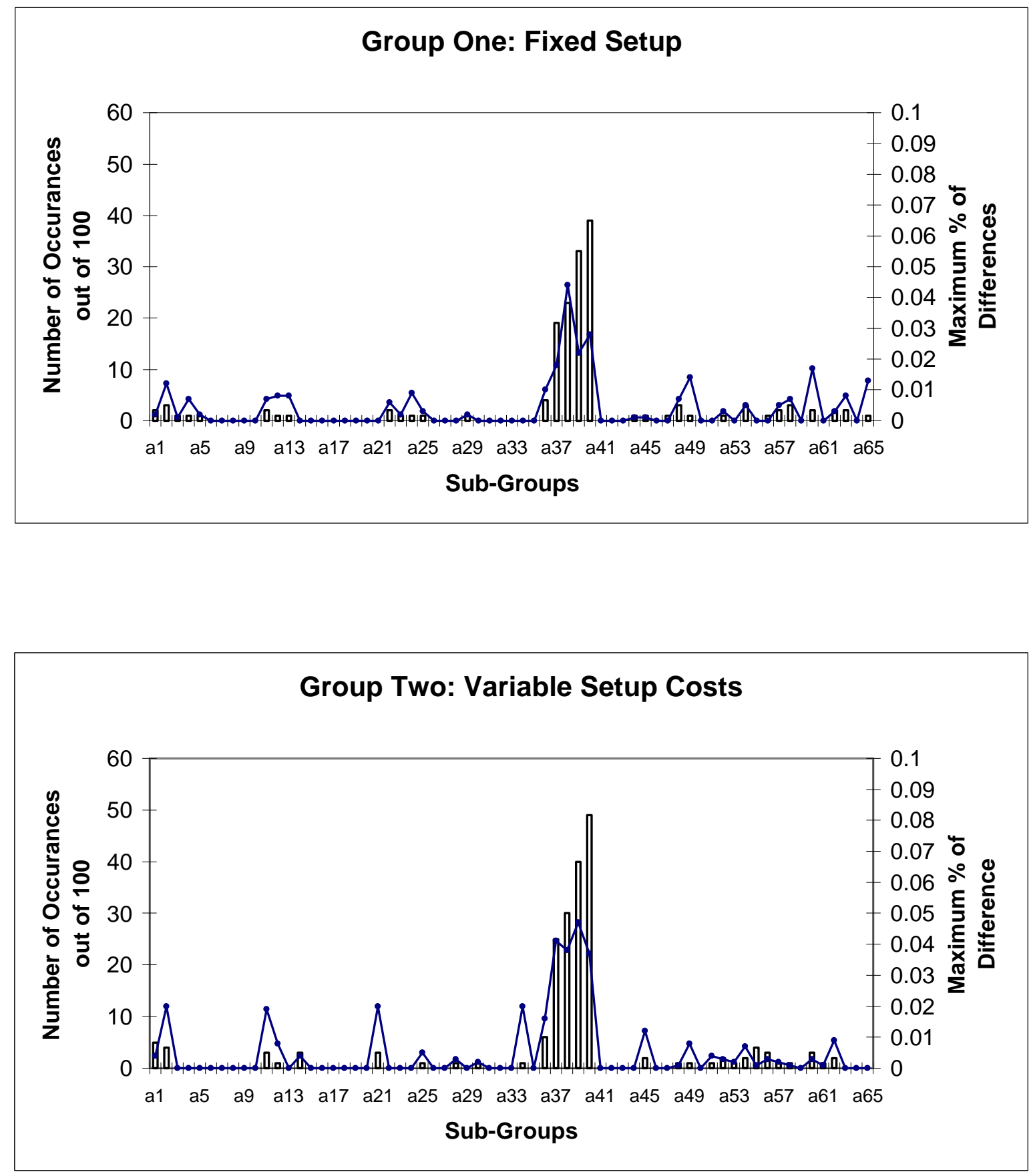

\section{Group Three: Fixed Production and Transportation Costs}

In all 4,500 instances in this group, the Shortest Path Algorithm produces the same solution as the Mixed Integer Program, i.e., the number of occurrences and the maximum \% of difference are 0 in all cases.

Figure 6. Comparing the Shortest Path and the MIP Solutions 


\section{A Solution Methodology for the Multi-Item, Multi-Facility Problem $(P)$}

We propose a Lagrangean Decomposition scheme for the solution of the multi-item, multifacility problem $(P)$ where the single-item problem $\left(P_{i}\right)$ is a subproblem. Lagrangean Decomposition, as described in Guignard and Kim (1987), has been applied to a variety of NPhard problems including multi-item single-facility lot-sizing problems (Thizy, 1991). A main advantage of Lagrangean Decomposition over the better known Lagrangean Relaxation is that the theoretical Lower Bound obtained from Lagrangean Decomposition is at least as tight as that from Lagrangean Relaxation. We start our exposition by first listing the mass balance constraints (1.2) explicitly:

$$
\begin{array}{lc}
\sum_{t=1}^{T} D_{t}^{i}=\sum_{j=1}^{J_{i}} f_{j}^{i} & \forall i \in N \\
f_{j}^{i}=\sum_{t=1}^{T} x_{j t}^{i} & \forall i \in N, j \in J_{i} \\
x_{j t}^{i}+I_{j t-1}^{i}=b_{j t}^{i}+I_{j t}^{i} & \forall i \in N,(j, t) \in A \\
\sum_{j=1}^{J_{i}} b_{j t}^{i}=D_{t}^{i} & \forall i \in N, t \in T
\end{array}
$$

If we assume that the system must return to its initial inventory at the end of the planning horizon, i.e., $I_{0}=I_{T}$, we may simplify the above mass balance constraints. From (1.2.3) and (1.2.4) we have,

$$
D_{t}^{i}=\sum_{j=1}^{J_{i}}\left(x_{j t}^{i}+I_{j t-1}^{i}-I_{j t}^{i}\right), \forall i, t, \text { thus } \sum_{t=1}^{T} D_{t}^{i}=\sum_{t=1}^{T} \sum_{j=1}^{J_{i}}\left(x_{j t}^{i}+I_{j t-1}^{i}-I_{j t}^{i}\right)
$$

but since $I_{0}=I_{T}$

$\sum_{t=1}^{T} I_{j t-1}^{i}=I_{0}+\sum_{t=1}^{T-1} I_{j t}^{i}=\sum_{t=1}^{T-1} I_{j t}^{i}+I_{T}=\sum_{t=1}^{T} I_{j t}^{i}$, so we can rewrite (1.2.5) as

$\sum_{t=1}^{T} D_{t}^{i}=\sum_{j=1}^{J_{i}} \sum_{t=1}^{T} x_{j t}^{i}, \forall i \in N$

Note that (1.2.6) implies constraints (1.2.1) and (1.2.2). This allows us to consider problem (P) with only two sets of balance constraints (1.2.3) and (1.2.4) since (1.2.1) and (1.2.2) will be satisfied automatically. Now, consider the objective function of $(\mathrm{P})$ :

Minimize $z=\sum_{t=1}^{T} \sum_{i=1}^{n} \sum_{j=1}^{J_{i}}\left(c_{j t}^{i} x_{j t}^{i}+K_{j}^{i} \delta_{j t}^{i}+h_{j t}^{i} I_{j t}^{i}+r_{j t}^{i} b_{j t}^{i}\right)$

Since $b_{j t}^{i}=x_{j t}^{i}+I_{j t-1}^{i}-I_{j t}^{i}$ (from (1.2.3)), the objective can be re-rewritten as follows:

Minimize $z=\sum_{t=1}^{T} \sum_{i=1}^{n} \sum_{j=1}^{J_{i}}\left(\left(c_{j t}^{i}+r_{j t}^{i}\right) x_{j t}^{i}+\left(h_{j t}^{i}-r_{j t}^{i}+r_{j t+1}^{i}\right) I_{j t}^{i}+K_{j}^{i} \delta_{j t}^{i}\right)$ 
If we set $\hat{c}_{j t}^{i} \equiv c_{j t}^{i}+r_{j t}^{i}$ and $\hat{h}_{j t}^{i} \equiv h_{j t}^{i}-r_{j t}^{i}+r_{j t+1}^{i}$ to be the generalized production and holding costs, the objective becomes:

Minimize $z=\sum_{t=1}^{T} \sum_{i=1}^{n} \sum_{j=1}^{J_{i}}\left(\left(\hat{c}_{j t}^{i} x_{j t}^{i}+\hat{h}_{j t}^{i} I_{j t}^{i}+K_{j}^{i} \delta_{j t}^{i}\right)\right.$

The basic idea of our decomposition is to separate the multicommodity flows problem $(P)$ into two subproblems: the first with the capacity and the mass balance constraints, but not the setup constraints, the second is a commodity-decomposable subproblem with the mass balance and the setup constrains. The latter defines separable single-item problems $\left(P_{i}\right)$, which has special structure as analyzed in Section 3. In this decomposition, the first subproblem is a linear program and the second is a collection of MIP problems. If we make use of the shortest path algorithm for each MIP (as a heuristic), all subproblems are easy to solve. To demonstrate this solution methodology we use a slightly simplified formulation of problem $(\mathrm{P})$ by dropping the first term in the objective (as above), and assuming that setup does not consume capacity (dropping (2.1)). We then restate the multi-facility production problem (P) with duplicated variables as follows:

(P’)

$$
\begin{array}{lc}
\text { Minimize } z=\sum_{t=1}^{T} \sum_{i=1}^{n} \sum_{j=1}^{J_{i}}\left(\left(\hat{c}_{j t}^{i} x_{j t}^{i}+\hat{h}_{j t}^{i} I_{j t}^{i}+K_{j}^{i} \delta_{j t}^{i}\right)\right. \\
\text { s.t. } \\
\sum_{i=1}^{n} \beta_{j t}^{i} x_{j t}^{i} \leq u_{j t} & \forall(j, t) \in A \\
x_{j t}^{i}+I_{j t-1}^{i}-I_{j t}^{i} \geq 0 & \forall i \in N,(j, t) \in A \\
\sum_{j=1}^{J_{i}}\left(x_{j t}^{i}+I_{j t-1}^{i}-I_{j t}^{i}\right)=D_{t}^{i} & \forall i \in N, t \in T \\
x_{j t}^{i}=x x_{j t}^{i} & \forall i \in N, j \in J, t \in T \\
I_{j t}^{i}=I I_{j t}^{i} & \forall i \in N, j \in J, t \in T \\
x x_{j t}^{i}+I I_{j t-1}^{i}-I I_{j t}^{i} \geq 0 & \forall i \in N,(j, t) \in A \\
\sum_{j i}^{J_{i}}\left(x x_{j t}^{i}+I I_{j t-1}^{i}-I I_{j t}^{i}\right)=D_{t}^{i} & \forall i \in N, t \in T \\
x_{j t}^{i} \leq M \delta_{j t}^{i} & \forall i \in N, j \in J, t \in T \\
x_{j t}^{i}, I_{j t}^{i} \geq 0 & \forall i \in N, j \in J, t \in T \\
x x_{j t}^{i}, I I_{j t}^{i} \geq 0 & \forall i \in N, j \in J, t \in T \\
\delta_{j t}^{i} \in(0,1) & \forall i \in N, j \in J, t \in T
\end{array}
$$


In the above formulation, we make copies of the variable $x_{j t}^{i}, b_{j t}^{i}$, and $I_{j t}^{i}$ as $x x_{j t}^{i}, b b_{j t}^{i}$, and $I I_{j t}^{i}$. We then use the copies to split the original constraints into two sets of constraints: $\{(4.1)$ (4.3),(4.7) $\}$ and $\left\{(4.2)^{\prime},(4.3)^{\prime},(4.6),(4.8),(4.9)\right\}$ plus the linking constrains (4.4)-(4.5). It should be clear that $(\mathbf{P}) \equiv\left(\mathbf{P}^{\prime}\right)$. We then separate $\left(\mathbf{P}^{\prime}\right)$ by relaxing the linking constraints and placing them in the objective function with Lagrangean multipliers $\lambda^{x}{ }_{i j t}$ and $\lambda_{i j t}^{I}$. This yields the following subproblems:

\section{Resource Subproblem:}

$$
\text { Minimize } \quad z_{1}=\sum_{t=1}^{T} \sum_{i=1}^{n} \sum_{j=1}^{J_{i}}\left(\left(\hat{c}_{j t}^{i}+\lambda_{i j t}^{x}\right) x_{j t}^{i}+\left(\hat{h}_{j t}^{i}+\lambda_{i j t}^{I}\right) I_{j t}^{i}\right) \equiv z_{1}(\lambda)
$$

s.t. (4.1), (4.2), (4.3), (4.7)

\section{n Product Subproblems:}

Minimize $\quad z_{2}=\sum_{i=1}^{n}\left(\sum_{t=1}^{T} \sum_{j=1}^{J_{i}}\left(-\lambda_{i j t}^{x} x x_{j t}^{i}-\lambda_{i j t}^{I} I I_{j t}^{i}+K_{j}^{i} q_{j t}^{i}\right)\right) \equiv \sum_{i=1}^{n} z_{2}^{i}(\lambda)$

s.t. $(4.2)^{\prime},(4.3)^{\prime},(4.6),(4.8),(4.9)$

Note that under the general framework of Lagrangean Decomposition, constraints (4.2) and (4.3) do not need to be duplicated for both subproblems, i.e., these constraints can be assigned to either subproblems. However, our computational experience indicates that as long as the added constraints do not add computational burden to the subproblems, constraint duplication improves the speed of convergence and yield better lower bounds since the solutions proposed by the subproblems tend to be similar. A lower bound to problem ( $\mathbf{P}^{\prime}$ ) given Largrangean multiplier set $\lambda$ is as follows:

$$
L B_{\lambda}\left(P^{\prime}\right)=v\left(z_{1}(\lambda)\right)+\sum_{i=1}^{n} v\left(z_{2}^{i}(\lambda)\right)
$$

Where $v($.$) denote the optimal value of the objective. Note that the resource subproblem$ is a linear program, and the product subproblem $z_{2}^{i}$ has similar structure as problem $\left(P_{i}\right)$ described earlier. From the lower bound solution, an upper bound for problem (P') can be generated using the following feasibility restoration routine: given the solution for the resource subproblem we add setups for the periods where production is nonzero, i.e., we set $q_{j t}^{i}$ to 1 whenever $x_{j t}^{i}>0$. We then calculate the objective function using the original cost function. This results in an upper bound for the original problem. The lower bound can be maximized by 
searching for the set of Largrangean multipliers $\lambda$ that maximize the Lagrangean dual. Both dual ascent and subgradient search methods can be used for this task. In this paper, we use the latter approach, which is summarized in the following Section 4.2. As we will demonstrate in the computational section, we can achieve solutions with very small duality gaps using the bounds and the search algorithm.

\subsection{Managerial Insights Related to the Decomposition}

Our choice of Lagrangean Decomposition is not purely motivated by computing. The decomposition of the multi-facility production model into a resource subproblem and multiple product subproblems has interesting managerial implications. As recognized by several researchers (c.f., Jörnsten and Leisten, 1994; Burton and Obel, 1984), mathematical decomposition often leads to insights for general modeling strategies or even new decision structures. The decomposition suggested earlier allows further analysis concerning modeling flexibility in the context of multi-facility manufacturing planning. Suppose we consider each product subproblem as a decision problem for a product manager and the resource subproblem as a decision problem for a production manager overseeing multiple facilities. Thus, the decomposition can be viewed as a decision system where product managers, each responsible for a product, compete for resource capacity available from manufacturing facilities. The production manager, on the other hand, represents the interests of efficiently allocating resources from multi-facilities to the products. Clearly the solutions proposed by the production manager $(x, I)$ do not agree with the collective solution proposed by the product managers $(x x, I I)$. A search based on Lagrangean multipliers essentially penalizes their differences, while adjusting the penalty vector iteratively. This process stops when the degree of disagreement (the duality gap) is acceptably low, or when further improvement is unlikely.

The above viewpoint is useful in evaluating the flexibility model $(P)$ represents. First, it should be clear that each product subproblem $\left(P_{i}\right)$ could be customized to represent the distinctive needs of each product. So long as its basic network structure is maintained there will be no additional computational burden. Similarly, as long as the resource subproblem remains a linear program, it can be customized with various facility submodels each reflecting the distinct production structure of a facility. However, a different constraint duplication strategy may be necessary when changes are made to the base model.

\subsection{The Subgradient Search Algorithm}

In this section, we summary the subgradient search algorithm used to adjust the Lagrangean multipliers. At each iteration $s$, we calculate Lagrangean multipliers using the following equations: 


$$
\begin{aligned}
& \lambda_{i j t}^{x, s+1}=\lambda_{i j t}^{x, s}+u^{s}\left(x_{j t}^{i, s}-x x_{j t}^{i, s}\right) \\
& \lambda_{i j t}^{I, s+1}=\lambda_{i j t}^{I, s}+u^{s}\left(I_{j t}^{i, s}-I I_{j t}^{i, s}\right)
\end{aligned}
$$

where

$$
u^{s}=\frac{\gamma_{s}\left(U B_{s}^{*}-\left(v\left(z_{1}\left(\lambda^{s}\right)\right)+\sum_{i=1}^{n} v\left(z_{2}^{i}\left(\lambda^{s}\right)\right)\right)\right.}{\sum_{t=1}^{T} \sum_{i=1}^{n} \sum_{j=1}^{J_{i}}\left(\left(x_{j t}^{i, s}-x x_{j t}^{i, s}\right)^{2}+\left(I_{j t}^{i, s}-I I_{j t}^{i, s}\right)^{2}\right)}
$$

$\gamma_{s}$ : a scalar set to 1 and reduced by half if the lower bound fails to improve after a fixed number of iterations

$U B^{*}$ : the best upper bound obtained up to iteration $s$

In our testing, we terminate the algorithm after a prespecified number of iterations. The best upper bound obtained at the end of the iterations provides the heuristic solution to the problem. We summarize the algorithmic steps as follows:

Step 1: Initialize $s, \lambda, u, \gamma$ and $U B^{*}$.

Step 2: Solve the resource and the product subproblems. Compute the lower bound $\left(v\left(z_{1}\left(\lambda^{s}\right)\right)+\sum_{i=1}^{n} v\left(z_{2}^{i}\left(\lambda^{s}\right)\right)\right.$ for current iteration, $s$.

Step 3: Compute an upper bound $U B_{\text {s }}$ from the optimal solution of the current resource subproblem Min $z_{1}\left(\lambda^{s}\right)$. If $U B_{s}<U B_{s-1}^{*}$, set $U B_{s}^{*} \leftarrow U B_{s}$.

Step 4: Update the multipliers using equations (4.10) and (4.11)

Step 5: Stop if a prespecified iteration limit is reached. Otherwise go to Step 2.

\section{Computational Testing}

\subsection{Effectiveness of Lagrangean Decomposition and Subgradient Search}

We implemented the subgradient search algorithm using the mathematical programming language AMPL with the CPLEX solver. The experiments are conducted on a Pentium-200 personal computer with 64Meg RAM. To test the effectiveness of the Largrangean decomposition and the subgradient search algorithm, we generate 450 test instances with 30 distinctly different problem characteristics. The product subproblems are solved using the CPLEX MIP solver. For each test instance we use 150 iterations of subgradient search and record the best lower and upper bounds at the end of the iteration to compute the duality gap. We first generate two sets of nominal case problems $(C 1)$ with 4 facilities and 6 periods. The first set assumes fixed transportation (FT) costs while the second set assumes variable transportation (VT) costs. The demand and cost parameters used for the nominal case is summarized in the footnotes of Table 1. As shown, the production, inventory and setup costs as 
well as item demands are randomly generated using Uniform distribution. To generate capacity we use the following procedure: we first calculate cumulative demands by adding the randomly generated demands of all items up to period $t$ for all $t \in T$. For the first period, we multiply the total demand for the period by some constant $(\geq 1)$. We then use this number as the total capacity available in the period and assign a fraction to each facility. For the coming periods, total capacities assigned for the previous time periods are subtracted from the cumulative demand of that period and then multiplied by some constant to generate the capacity. Using this procedure, we may generate relatively challenging (but feasible) test problems with tight capacity constraints. For the nominal test problems theses constants are set at 1.3. Fifteen replications are assigned to each case. We then alter the nominal cases by changing problem characteristics and sizes to generate 14 additional cases (C2-C15) each including the fixed and variable transportation (FT and VT) cases and each are repeated for 15 replications. This results in 450 test instances and the average duality gaps are summarized in Table 1.

Table 1. Duality Gaps for 450 Test Instances over Various Cost Structures and Problem Sizes

\begin{tabular}{|l|l|l|l|l|l|l|l|l|l|l|l|l|l|l|l|}
\hline$\%$ & C1 & C2 & C3 & C4 & C5 & C6 & C7 & C8 & C9 & C10 & C11 & C12 & C13 & C14 & C15 \\
\hline FT & 4.07 & 1.72 & 7.25 & 1.82 & 0.91 & 4.36 & 2.27 & 3.73 & 4.93 & 0.84 & 2.09 & 3.10 & 12.3 & 7.05 & 25.9 \\
\hline VT & 4.02 & 1.39 & 6.51 & 1.87 & 0.94 & 4.00 & 2.54 & 2.78 & 5.13 & 0.84 & 2.01 & 2.64 & 12.4 & 6.83 & 25.9 \\
\hline
\end{tabular}

Duality gaps are calculated as $(U B-L B) / U B * 100 \%$ each table entry is averaged over 15 replications C1- Nominal Case: Demand U[0,200], Setup cost U[1500,3500], Holding cost U[5,15], Production cost $\sim$ U [5,15], 30 items, 4 facilities, 6 periods, Capacity tightness factor $=1.3$, Transportation cost: 10 for FT, U [5,15] for VT

The following cases represent variations from the nominal cases by the indicated factor(s)

C2- Low setup where setup cost $\sim$ Uniform $[0,1000]$

C3- High Setup where setup cost Uniform [4000,8000]

C4- High Production Costs where Production cost Uniform [40,80]

C5- Very High Production Cost where Production cost Uniform [100,200]

C6- Lumpy demand: expected demand is 100 but there is a 0.3 probability that demand is 0

C7- Low demand variability where Demand $~$ Uniform $[50,150]$

C8- Loose capacity: capacity tightness factor is set at 1.8

C9- Tight capacity: capacity tightness factor is set at 1.15

C10- Number of facilities $=1$

C11- Number of facilities $=2$

C12- Number of facilities $=3$

C13- Number of items $=10$

C14- Number of items $=20$

C15- Projected worst case: Lumpy demand, High setup cost, Tight capacity, number of items $=10$

For simplicity, we assume $\beta_{j t}^{i}$ (the consumption rate of facility $j$ 's resoure by item $i$ at period $t$ ) is equal to 1 . We also assume the starting and ending inventory to be zero. For most of the test problems lower bound increases significantly in the first 20 iterations whereas the upper bound improves slowly. There appears to be a strong correlation between the quality of the lower and the upper bounds, i.e., when the lower bound obtained is tight, the upper bound 
restored from the lower bound solution is also of higher quality. We observed a quite consistent convergence pattern throughout all test problems. Convergence typically occurs quite early resulting in a very small duality gap. We summarize the observations from Table 2 as follows:

1. As shown in the table, setup cost appears to have a significant effect on the duality gap. Low setup instances has an average gap of $1.72 \%$ and $1.39 \%$ compared to $7.25 \%$ and $6.51 \%$ for the high setup instances. This result is not surprising since an increased setup costs widen the gap between the resource subproblem (which is an LP ignoring the setup cost) and the product subproblems. On the other hand, since the original problem is a mixed integer program with binary setup variables, as the setup costs increase the problem behave closer to a combinatorial problem then an LP.

2. The number of facilities appears to have an effect on the duality gap as well. Comparing cases $\mathrm{C} 1$, and $\mathrm{C} 10-\mathrm{C} 12$. As the number of facilities increases we observe a monotonic increase in duality gap as well. This result is useful in that making alternative facility production decisions is an unique feature of our model. The results suggest that the added dimension has a noticeable effect on the difficulty of the problem. On the other hand, it also shows that the proposed algorithm is quite effective in solving the traditional single-facility problems $(\mathrm{C} 10)$.

3. The effect of capacity levels is much less pronounced. This may due to the fact that the capacity generation procedure produces relatively tight capacity in all instances. Since the difference between non-capacitated and capacitated lot sizing models is well known, we did not make an attempt to further loosen the capacity.

4. Increasing the number of items appears to have an effect on the duality gap as well. Problems with a larger number of items appear to have a smaller duality gap.

\subsection{Effectiveness of the Shortest Path Algorithm as a Subproblem Heuristic}

The results in Table 1 are produced by Lagrangean Decomposition using subgradient search where each single-item, multi-facility subproblem is solved as an MIP. As demonstrated in Section 3.4, the shortest path algorithm can be an effective heuristic for the single-item subproblem (the solution never deviate from the optimal by more than $0.5 \%$ ). We are interested in the effectiveness of the shortest path algorithm as a heuristic in the context of subgradient search. The potential saving in computer time is significant as the subgradient search algorithm must solve $|N|$ single-item subproblem in each iteration, this results in up to 3,000 calls to the subproblem (i.e., 20 items, and 150 iterations). However, it should be recognized that the subgradient search method might not work properly when the shortest path algorithm is used to solve the subproblem. This is the case when the shortest path algorithm produces suboptimal 
solutions to the (capacity-relaxed) subproblem and the solution values (of the relaxed subproblem) exceed the optimum of the original (capacitated) problem. In Table 2, we compare the bounds generated for the multi-item multi-facility problem (P') when MIP and the shortest path algorithm are used to solve the single-item subproblems.

Table 2. Comparing the Quality of Bounds for the Multi-Item Problem when the SingleItem Problems are solved by the MIP and the Shortest Path Algorithm

\begin{tabular}{|c|c|c|c|c|c|c|c|c|c|c|c|c|c|c|c|}
\hline & C1 & $\mathrm{C} 2$ & C3 & $\mathrm{C} 4$ & C5 & $\mathrm{C} 6$ & $\mathrm{C} 7$ & $\mathrm{C} 8$ & $\mathrm{C9}$ & $\mathrm{C} 10$ & C11 & $\mathrm{C} 12$ & $\mathrm{C} 13$ & $\mathrm{C} 14$ & C15 \\
\hline $\begin{array}{l}\# \text { of times* } \\
U B(S P)<U B(M I P)\end{array}$ & 14 & 9 & 13 & 12 & 13 & 10 & 15 & 14 & 8 & 11 & 13 & 12 & 14 & 11 & 12 \\
\hline $\begin{array}{l}\text { \% difference in UB } \\
\text { values }\end{array}$ & 0.40 & 0.43 & 1.02 & 0.25 & 0.24 & 0.97 & 0.49 & 0.51 & 0.57 & 0.20 & 0.00 & 0.00 & 1.28 & 1.04 & 1.96 \\
\hline $\begin{array}{l}\text { \# of times } \\
U B(S P)>\cup B(M I P)\end{array}$ & 1 & 6 & 2 & 3 & 2 & 5 & 0 & 1 & 7 & 4 & 2 & 3 & 1 & 4 & 3 \\
\hline $\begin{array}{l}\text { \% difference in UB } \\
\text { values }\end{array}$ & 2.65 & 0.18 & 0.03 & 0.45 & 0.04 & 0.25 & 0.00 & 0.45 & 0.64 & 0.14 & 0.03 & 0.34 & 0.04 & 0.59 & 0.61 \\
\hline $\begin{array}{l}\# \text { of times } \\
U B(S P)=U B(M I P)\end{array}$ & 1 & 0 & 0 & 0 & 0 & 0 & 0 & 0 & 0 & 0 & 13 & 12 & 0 & 0 & 0 \\
\hline $\begin{array}{l}\text { \# of times } \\
L B(S P)>L B(M I P)\end{array}$ & 7 & 4 & 10 & 4 & 4 & 5 & 4 & 6 & 3 & 0 & 0 & 1 & 7 & 7 & 5 \\
\hline $\begin{array}{l}\% \text { difference in } L B \\
\text { values }\end{array}$ & 0.03 & 0.18 & 0.04 & 0.01 & 0.01 & 0.04 & 0.03 & 0.01 & 0.06 & 0.00 & 0.00 & 0.01 & 0.03 & 0.08 & 0.06 \\
\hline \begin{tabular}{|l} 
of times $^{*}$ \\
$L B(S P)<L B(M I P)$ \\
\end{tabular} & 5 & 7 & 4 & 3 & 6 & 6 & 3 & 5 & 11 & 0 & 0 & 0 & 7 & 4 & 9 \\
\hline$\%$ difference in $L B$ values ${ }^{* *}$ & 0.02 & 0.02 & 0.03 & 0.02 & 0.01 & 0.02 & 0.01 & 0.02 & 0.04 & 0.00 & 0.00 & 0.00 & 0.06 & 0.03 & 0.23 \\
\hline $\begin{array}{l}\text { \# of times } \\
L B(S P)=L B(M I P)\end{array}$ & 3 & 4 & 1 & 8 & 5 & 4 & 8 & 4 & 1 & 15 & 15 & 14 & 1 & 4 & 1 \\
\hline
\end{tabular}

* the number of occurrences out of 15 instances

** averaged over the number of occurrences in the cells above cases $\mathrm{C} 1-\mathrm{C} 15$ are equivalent to cases defined in Table 1

It should be evident that when the shortest path algorithm is used to solve the single-item subproblem, it produces nearly identical lower and upper bounds for the multi-item problem. The percentage difference in lower bounds produced by shortest path and MIP never exceeds 1\% in all but 2 cases (out of 450 instances), in most cases, the difference is less than $0.02 \%$. Even in the worst cases (case C15) where the shortest path is expected to generate different solutions than MIP, the actual difference averaged at $0.23 \%$. To further test these worst cases, we generate additional five classes of test problems (C16-C20) by intentionally increasing the transportation costs, lower the setup costs, and the combinations of the two. These additional results are given in Table 3. As can be seen from the table, the difference in bound quality between shortest path and MIP is still less than $0.29 \%$ on average. However, MIP does generate better upper bounds and tighter lower bounds in these cases. 
Table 3. Comparing the Quality of Bounds for Specially Generated Worst Cases

\begin{tabular}{|c|c|c|c|c|c|}
\hline & C16 & C17 & C18 & C19 & $\mathrm{C} 20$ \\
\hline $\begin{array}{l}\text { \# of times } \\
U B(S P)<U B(M I P)\end{array}$ & 12 & 0 & 2 & 3 & 1 \\
\hline$\%$ difference in UB values* & 0.29 & 0.00 & 0.03 & 0.04 & 0.01 \\
\hline \# of times ${ }^{*} \cup B(S P)>U B(M I P)$ & 3 & 15 & 10 & 10 & 14 \\
\hline$\%$ difference in UB values ${ }^{* *}$ & 0.16 & 0.24 & 0.08 & 0.05 & 0.09 \\
\hline $\begin{array}{l}\text { \# of times } \\
U B(S P)=U B(M I P)\end{array}$ & 0 & 0 & 3 & 2 & 0 \\
\hline \# of times ${ }^{*} L B(S P)>L B(M I P)$ & 0 & 0 & 0 & 2 & 0 \\
\hline $\begin{array}{l}\% \text { difference in } L B \\
\text { values }\end{array}$ & 0.00 & 0.00 & 0.00 & 0.03 & 0.00 \\
\hline \# of times ${ }^{*} L B(S P)<L B(M I P)$ & 15 & 15 & 15 & 12 & 15 \\
\hline$\%$ difference in $L B$ values ${ }^{* *}$ & 0.10 & 0.20 & 0.06 & 0.06 & 0.12 \\
\hline $\begin{array}{l}\text { \# of times } \\
L B(S P)=L B(M I P)\end{array}$ & 0 & 0 & 0 & 1 & 0 \\
\hline
\end{tabular}

the number of occurrences out of 15 instances

** averaged over the number of occurrences in the cells above

C16: High Transportation Costs $\sim$ Uniform[40,80]

C17: Very High Transportation Costs $\sim$ Uniform[100,200]

C18: Low Setup Uniform [0,1000], High Transportation Costs Uniform[40,80]

C19: Low Setup Uniform [0,1000], High Transportation Costs Uniform[40,80], Lumpy Demand

C20: Low Setup Uniform [0,1000], Very High Transportation Costs Uniform[100,200]

Another point of interest is during the subgradient search, how often does the shortest path solution deviate from the solution generated by MIP. The statistics collected on this particular measure is given in Figure 7.

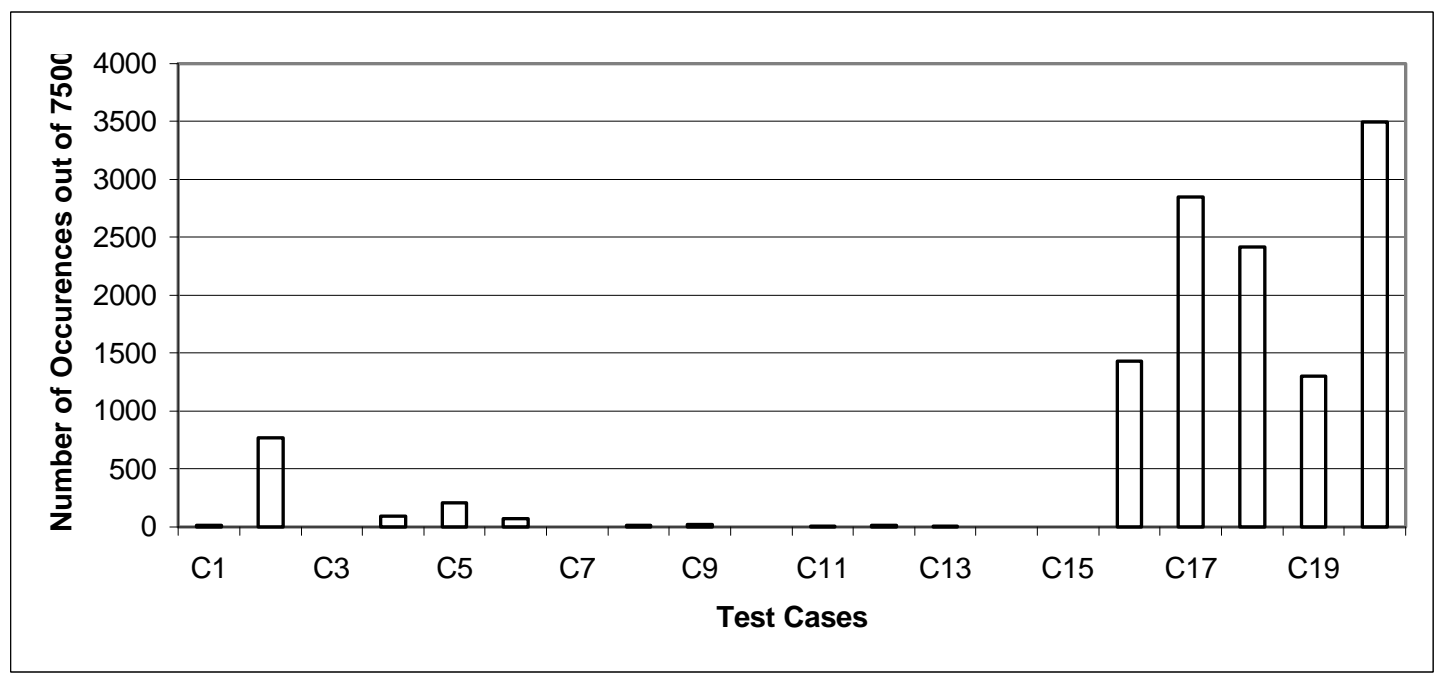

Figure 7. The average frequency when the shortest path solution deviates from MIP during the subgradient search $(150$ iterations $\times 50$ Items $=7500$ instances under each test case, averaged over 15 replications) 

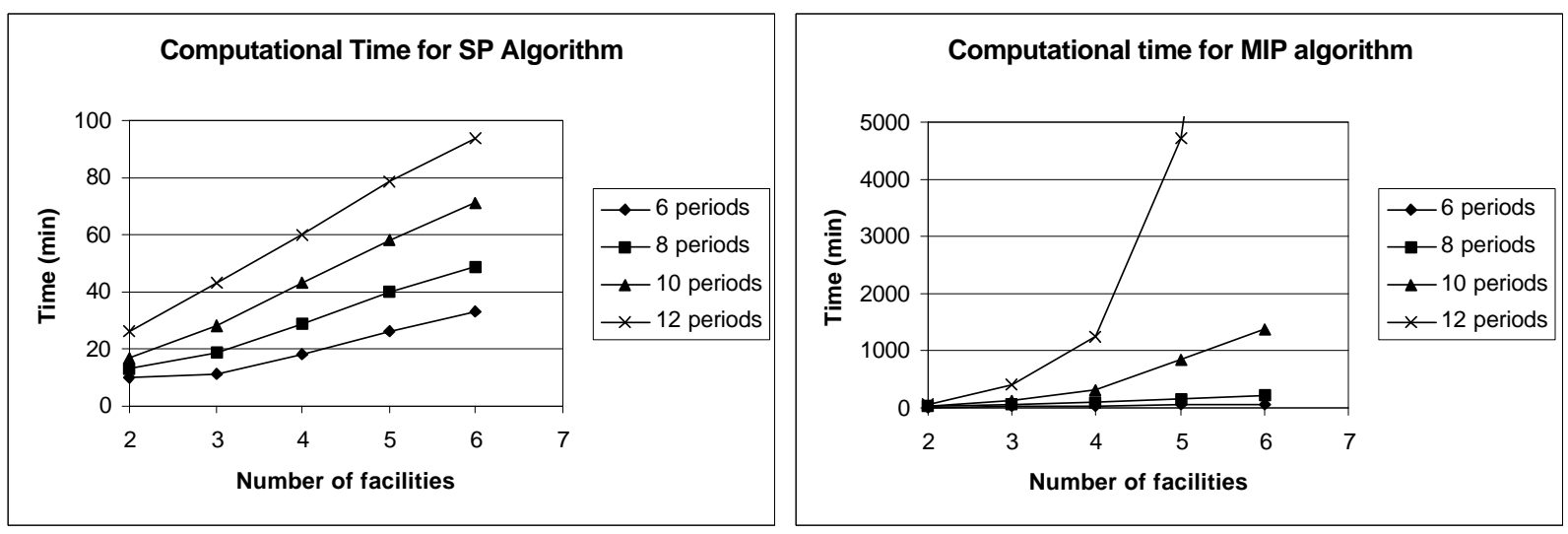

Figure 8. Comparison Between Shortest Path Algorithm and MIP in the Multi-item problem (each data point represent 150 iterations of subgradient search)

A main incentive of studying the single-item, multi-facility shortest path algorithm is the potential computational saving when solving the multi-item problem. We conduct an additional set of experiments that compare the computational efficiency of the shortest path algorithm against MIP. While it is obvious that the shortest path algorithm will take much less time in each single-item problem, we are interested to know the real saving in the context of the multi-item, multi-facility problem, and the effect of increasing the number of facilities and number of periods. We generate 20 multi-item problems with 2 , $3,4,5$, or 6 facilities, and $6,8,10$ or 12 periods. Each problem is solved using subgradient search with 150 iterations implemented in AMPL/CPLEX. The total computer time is recorded in CPU minutes, and the results are summarized in Figure 8. As shown in the figure, when the shortest path algorithm is used for the single item subproblem, the computer time increase in a near linear fashion as the number of facilities increase. On the other hand, when we solve an MIP for each subproblem, the computer time increases much more dramatically. An exponential growth in computer time can be observed when testing on the 12-period problem, where we can only solve up to 5 facilities with the 5,000 CPU-minute limit. For problems that are solvable using the MIP subproblem we observe a much sharper increase in CPU time as the number of periods increases. This is consistent with the complexity insights given in Theorem 4 .

\section{Acknowledgement:}

This research is supported, in part, by National Science Foundation grant DMI-9634808 and a grant from Lucent Technologies. We appreciate insightful comments from Professors Thomas Liebling and George Nemhauser on an earlier version of the formulation. 


\section{References:}

Afentakis, P., B. Gavish and U. Karmarker (1984), "Computational Efficient Optimal Solutions to the Lot-Sizing Problems in Multistage Assembly Systems," Management Science, 30, 222-39.

Arntzen, Bruce C, Gerald G. Brown, Terry P. Harrison, and Linda L. Trafton (1995) "Global Supply Chain Management at Digital Equipment Corporation." Interfaces 25: 1 JanuaryFebruary 1995, pp. 69-93.

Assad, A.A., (1980) “Models for Rail Transportation,” Transportation Research, 14A, 205-220.

Baker, K.R. (1993) "Requirements Planning" in Logistics of Production and Inventory, Handbooks in Operations Research and Management Science, Volume 4, ed. S.C. Graves, et al, Elsevier Science Publishers, Amsterdam, The Netherlands.

Bahl, H.C., Ritzman, L.P., and Gupta, J.N.D. (1987), "Determining Lot Sizes and Resource Requirements: A Review," Operations Research, Vol. 35, pp. 329-345.

Billington, Peter J., John O. McClain, and L. Joseph Thomas. (1983). "Mathematical Programming Approaches to Capacity-Constrained MRP Systems: Review, Formulation and Problem Reduction." Management Science, vol. 29, no. 10, October, pp 1126-1141.

Blackburn, J., and R. Millen (1982). "Improved Heuristics for Multi-Stage Requirements Planning Systems." Management Science, vol. 28, no. 1, pp. 44-56.

Burton, R.M., and Obel, B., (1984), Designing Efficient Organizations, North Holland, Amsterdam.

Carlson, Robert C., and Candace A. Yano (1983) "Safety Stocks in MRP - Systems with Emergency Setups for Components" Management Science, vol. 32, no. 4, April, pp 403-412.

Cohen, Morris A., and Hau L. Lee. (1988) "Strategic Analysis of Integrated ProductionDistribution Systems: Models and Methods." Operations Research, vol. 36, no. 2, pp 216-228.

and _ (1989) "Resource Deployment Analysis of Global Manufacturing and Distribution Networks." Journal of Manufacturing and Operations Management, no. 2, pp 81104.

Davis, Tom. (1993) "Effective Supply Chain Management." Sloan Management Review, Summer, pp 35-46.

Dreyfus, S.E. and R. A. Wagner (1972), “On the Steiner Problem in Graphs," Networks, 1, 195207. 
Ertogral, K. and S.D. Wu (1999), "Auction Theoretic Coordination of Production Planning in the Supply Chain," IIE Transactions, Special Issue on Design and Manufacturing: Decentralized Control of Manufacturing Systems, forthcoming.

Federgruen, A. and M. Tzur (1981), "A Simple Forward Algorithm to Solve General Dynamic Lot Sizing Models with n Periods in $O(n$ long $n)$ or $O(n)$ Time," Management Science, 27, 1-18.

Goyal, S.K. and Gunasekaran, A., (1990), "Multi-Stage Production-Inventory Systems," European Journal of Operational Research, Vol 46, pp. 1-20.

Graves, S.C., et al., (eds.) (1992) Handbooks in Operations Research and Management Science, Volume 4, Logistics of Production and Inventory. Amsterdam; Elsevier Science Publishers.

Guignard, M. and Kim, S. (1987), "Lagrangean Decomposition: A Model Yielding Stronger Lagrangean Bounds," Mathematical Programming, Vol. 39, pp. 215-228.

Gupta, S.M. and L Brennan. (1995) "MRP systems under supply and process uncertainty in an integrated shop floor control environment." International Journal of Production Research, vol. 33 , no. 1 , pp 205-220.

Hahm, Juho, and Candace Arai Yano, (1995a)"The Economic Lot and Delivery Scheduling Problem: the Common Cycle Case." IIE Transactions 27, 113-125.

and (1995b), "The Economic Lot and Delivery Scheduling Problem: Models for Nested Schedules." IIE Transactions 27, 126-139.

Jörnsten, K.O., Näsberg, M. and Smeds, P.A. (1986), "Variable Splitting: A New Lagrangean Relaxation Approach to Some Mathematical Programming Models," Technical Report LiTHMAT-R-85-04, Department of Mathematics, Linköping Institute of Technology, Sweden.

Jörnsten, K.O., and Leistern, R. (1994), "Aggregation and Decomposition for Multi-Divistional Linear Programs," European Journal of Operational Research, Vol. 72, 175-191.

Kennington, J.L. and R.V. Helgason (1980), Algorithms for Network Programming, WileyInterscience, New York.

Kimms, A. (1997), Multi-Level Lot Sizing and Scheudling, Physica-Verlag, Heidelberg, Germany.

Lee, Hau, P. Padmanabhan, Seungjin Whang (1995), "Information Distortion in a Supply Chain: The Bullwhip Effect." Working Paper, Stanford University, April, 1995.

Liebling, Thomas (1999), private communications.

Millar, H.H. and Yang, M. (1993), "An Application of Lagrangean Decomposition to the Capacitated Multi-Item Lot Sizing Problem,” Computers Ops. Res., Vol. 20, pp. 409-420. 
Sterman, John D. (1989) "Modeling Managerial Behavior: Misperceptions of Feedback in a Dynamic Decision Making Experiment." Management Science, Vol. 35, no. 3, March.

Tayur, S., R. Ganeshan and M. Magazine edited (1999) Quantitative Models for Supply Chain Management, Kluwer's International Series, Kluwer Academic Publishers, Norwell, MA.

Tempelmeir, H. and M. Derstroff (1996), "A Lagrangean Based Heuristic for Dynamic Multilevel Multiitem Constrained Lotsizing with Setup Times," Management Science, Vol 42, pp. 738-757.

Thizy, J.M., (1991), "Analysis of Lagrangian Decomposition for the Multi-Item Capacitated Lot-Sizing Problem," INFOR, Vol. 29, pp. 271-283.

Vidal, C.J. and M. Goetschalckx (1997), "Strategic Production-Distribution Models: A Critical Review with Emphasis on Global Supply Chain Models,” EJOR, 98, 1-18.

Wagelmans, A., S. Van Hoesel and A. Kolen (1992), "Economic Lot Sizing: An O(n log n) Algorithm that Runs in Linear Time in the Wagner-Within Case," Operations Research, Vol 40, 145-155. 


\section{Appendix}

\section{Proof for Theorem 1.}

It is easy to verify that problem $\left(P_{i}\right)$ has Leontief structures. The setup cost $K_{j}^{i}$ can be incorporated into the production $\operatorname{cost} C_{j t}^{i}$ as a fixed charge function as below:

$$
C_{j t}^{i}=\begin{array}{cl}
c_{j t}^{i} x_{j t}^{i}+K_{j}^{i} & \text { if } x_{j t}^{i}>0 \\
0 & \text { if } x_{j t}^{i}=0
\end{array}
$$

Other constraints are linear while the objective function is concave. Thus model $\left(P_{i}\right)$ has the following features: all nonnegative variables $x, I, b$, appear exactly once with a positive $(+1)$ coefficient; in all other occurrences they have a negative $(-1)$ coefficient. It follows that if more than one variable appears with a positive coefficient in the same constraints, then only one of these variables can be positive in the optimal solution, which results in the following conditions:

$$
b_{j t}^{i} b_{k t}^{i}=0 \quad \text { for } t=1 . . T, j=1 . . J_{i}, k=1 . . J_{i},
$$

This condition states the non-splitting property.

Similarly, the Leontief structure states the following:

$x_{j t}^{i} I_{j t-1}^{i}=0, \forall i, j, t$, or no production of item $i$ will take place when there is inventory in the same facility.

\section{Prooffor Theorem 2.}

We first state the uncapacitated single-item production-planning problem over alternative facilities by writing out the mass balance constraints. We concentrate on the problem where inventory holding costs $h$ are not restricted in sign, we call this problem $U C A P-N$.

$$
\begin{array}{ll} 
& \text { Minimize } \sum_{t=1}^{T} \sum_{j=1}^{J_{i}}\left(c_{j t} x_{j t}+K_{j} \delta_{j t}+h_{j t} I_{j t}\right) \\
& \text { s.t. } \\
& x_{j t}+I_{j t-1}-I_{j t}=b_{j t}, \quad \forall j \in J_{i}, t \in T \\
(\boldsymbol{U C A P}-N) \quad & \sum_{j=1}^{J_{i}} b_{j t}=D_{t}, \quad \forall t \in T \\
& x_{j t} \leq M \delta_{j t}, \quad \forall j \in J_{i}, t \in T \\
& x_{j t}, I_{j t} \geq 0, \quad \forall j \in J_{i}, t \in T \\
& \delta_{t} \in\{0,1\}
\end{array}
$$

To show that $U C A P-N$ is $N P$-complete we will show that the uncapacitated facility location ( $U F L$ ) problem can be reduced to $U C A P-N$. Specifically, we will show that for any given instance of the $N P$-complete problem $U F L$, there is a corresponding instance of $U C A P-N$. The decision version of $U F L$ is given as follows: 
(Uncapacitated Facility Location) There are $n$ demand points and $m$ facilities. Each demand point can be assigned to one of the several facilities (some facilities may not be possible for a specific demand point). When a demand point $i$ is assigned to facility $j$ (when $y_{j i}$ is set to 1), a demand assignment cost $d_{j i}$ incurs. It is necessary that all demand points be assigned to some facilities. When one or more demand points are assigned to a facility $j$ there is a cost, $f_{j}$ to open the facility, otherwise there is no cost. Given the above conditions, is there an assignment of demand points to facilities such that the total cost is lower than $\kappa$.

For convenience, we also state the optimization version of the $U F L$ problem as follows:

$$
\begin{aligned}
\text { Minimize } \quad z & =\sum_{i=1}^{n} \sum_{j=1}^{m} d_{j i} y_{j i}+\sum_{j=1}^{m} f_{j} \chi_{j} \\
\text { s.t. } \quad \sum_{j=1}^{m} y_{j i} & =1 \quad \forall i \\
\sum_{i=1}^{m} y_{j i} & \leq \chi_{j} \quad \forall j \\
y_{j i}, \chi_{j} & \in(0,1) \quad \forall i, j
\end{aligned}
$$

For a given instance of the $U F L$ problem we construct the following $U C A P-N$ instance: Construct $T=n+1$ periods including an initial period $s$ before period 1 so that each demand point $1 \ldots n$ corresponds to a planning period in $U C A P-N$. Construct $J_{i}=m$ facilities such that a facility in $U F L$ correspond to a facility in $U C A P-N$. Define set up cost $K_{j}$ for facility $j$ according to facility opening cost $f_{j}$. Set $c_{j s}=0$ and $c_{j t}=M$ for $t=1, \ldots, n$, where $M$ is an arbitrary large number. Set inventory holding costs $h$ and demands $D$ according to the demand assignment cost $d_{j i}$ as follows:

$$
\begin{array}{cccc}
h_{1 s} D_{1}=d_{11} & h_{2 s} D_{1}=d_{21} & \cdots & h_{m s} D_{1}=d_{m 1} \\
\left(h_{1 s}+h_{11}\right) D_{2}=d_{12} & \left(h_{2 s}+h_{21}\right) D_{2}=d_{22} & \cdots & \left(h_{m s}+h_{m 1}\right) D_{2}=d_{m 2} \\
\vdots & \vdots & & \vdots \\
\left(h_{1 s}+h_{11}+\ldots+h_{1, n-1}\right) D_{n}=d_{1 n} & \left(h_{2 s}+h_{21}+\ldots+h_{2, n-1}\right) D_{n}=d_{2 n} & \cdots & \left(h_{m s}+h_{m 1}+\ldots+h_{m, n-1}\right) D_{n}=d_{m n}
\end{array}
$$

Or more generally, $\left(\sum_{\tau=s}^{t-1} h_{j \tau}\right) D_{t}=d_{j t} \quad$ for $t=1, \ldots, n$.

Further, set $D_{s}=0$ and $I_{0}=0$. In the case where facility $j$ can not be used to satisfy the demand of point $i$, i.e., when $d_{j i}=\infty$ in a $U F L$ instance, set $d_{j i}$ to an arbitrary large number $M$. Thus, so long as the holding cost $h_{j t}$ is not restricted in sign, it is always possible to construct an instance of $h_{j t}$ and $D_{t}$ that satisfies all equations above. This and the above parameter setting allow us to construct an $U C A P-N$ (with parameters $c, h, K, D$ ) instance given any $U F L$ instance (with parameters $d, f$ ).

Now consider the solution of this specially constructed $U C A P-N$ instance. From Theorem 1 (the non-splitting property) we know that there exists an optimal solution to $U C A P-N$ 
where item $i$ 's demand in period $t$ is produced in exactly one of the $J_{i}$ facilities. Since $c_{j s}=0$ and $c_{j t}=M$ for $t=1, \ldots, n$, production will only take place in period $s$, at facility $j$. Demands in periods 1 to $n$ are satisfied completely by inventory carried over from period $s$, i.e., $x_{j s}=\sum_{t=1}^{n} D_{t} \delta_{j t}, \forall j$, and $I_{j t}=\sum_{\tau=t+1}^{n} D_{\tau} \delta_{j \tau}, \forall j, t$

Thus, the optimal solution to the $U C A P-N$ instance has a corresponding $U F L$ solution as follows:

(i) A production setup corresponds to facility opening, i.e., $\left\{\begin{array}{cc}\chi_{j}=1 \text { if } \sum_{t=s}^{n-1} \delta_{j t} \geq 1 \\ \chi_{j}=0 & \text { Otherwise }\end{array}\right.$

(ii) Production setup costs correspond to facility opening $\operatorname{cost} f_{j}$, for each $\chi_{j}=1$, i.e.,

$\sum_{t=s}^{n-1} \sum_{j=1}^{m} K_{j} \delta_{j t}=\sum_{j=1}^{m} f_{j} \chi_{j}$

(iii) Total holding costs correspond to demand assignment costs (from eq. (1)(2)), i.e.,

$\sum_{t=s}^{n-1} \sum_{j=1}^{m}\left(h_{j t} I_{j t}\right)=\sum_{t=s}^{n-1} \sum_{j=1}^{m}\left(h_{j t}\left(\sum_{\tau=t+1}^{n} D_{\tau} \delta_{j \tau}\right)\right)=\sum_{t=1}^{n} \sum_{j=1}^{m}\left(\left(\sum_{\tau=s}^{t-1} h_{j \tau}\right) D_{t}\right) \delta_{t}=\sum_{i=1}^{n} \sum_{j=1}^{m} d_{j i} y_{j i}$

In summary, the optimal value of the specially constructed $U C A P-N$ instance produces optimal value of the $U F L$ instance, i.e., in an optimal $U C A P-N$ solution,

$\sum_{t=s}^{n} \sum_{j=1}^{m}\left(c_{j t} x_{j t}+K_{j} \delta_{j t}+h_{j t} I_{j t}\right)=\sum_{i=1}^{n} \sum_{j=1}^{m} d_{j i} y_{j i}+\sum_{j=1}^{m} f_{j} \chi_{j}$

Thus, if the optimal solution of the UCAP-N instance has a total cost less than $\kappa$, then there is a corresponding UFL solution that has a total cost less then $\kappa$ (a yes answer to the decision problem). Otherwise, there is no assignment of demands to facilities with total cost lower than $\mathrm{\kappa}$ (a no answer).

Since $U F L$ is known to be $N P$-complete, this proves that $U C A P-N$ is also $N P$-complete.

\section{Proof of Corollary 1.}

The proof for this corollary can be done by a simple observation as follows: if all $D_{t}$ 's are constants in equation (1) above, we can still construct an $U C A P-N$ instance by finding $h_{j t}$ 's. The rest remains the same as the standard case.

\section{Proof of Corollary 2.}

To proof that when the setup cost $\delta_{j t}$ is constant across facilities $U C A P-N$ remains $N P$ Complete we only need to show that $U F L$ with constant facility opening cost $f_{j}$ is $N P$ Complete. We will show that the vertex cover $(V C)$ problem can polynomially reduced to $U F L$. For a given vertex cover instance, we construct an $U F L$ instance as follows: associate each edge in $V C$ to a demand point, and each vertex to a facility. When an edge 
is (not) incident to a vertex, the corresponding demand point can (not) be assigned to a facility (i.e., there is an edge $(i, j)$ between the demand point and the facility in $U F L$ ). Set $d_{j i}=0$ for all edges present and set facility opening $\operatorname{cost} f_{j}$ to a constant 1 for all facilities $j$. It is easy to verify that a solution to this specially constructed $U F L$ instance correspond to a solution to the original $V C$ instance.

Therefore, $U F L$ with constant facility opening cost is $N P$-complete, thus $U C A P-N$ with constant setup cost is NP-Complete.

\section{Proof of Theorem 3.}

The proof is similar to that of Theorem 2. It is therefore sufficient to outline the reduction from Uncapacitated Facility Location $(U F L)$ to $\left(P^{\prime}{ }_{i}\right)$. For a given instance of $U F L$ problem we may construct a $\left(P_{i}^{\prime}\right)$ instance as follows:

\begin{tabular}{|l|l|}
\hline \multicolumn{1}{|c|}{ Given an UFL instance $\ldots$} & \multicolumn{1}{c|}{ Construct a $\left(P_{i}^{\prime}\right)$ instance such that } \\
\hline Demand points $(i), i=1, \ldots, n$ & Periods $(t), t=1, \ldots, n$ \\
\hline Facilities $(j), j=1, \ldots, m$ & Facilities $(j), j=1, \ldots, m$ \\
\hline Facility opening cost $f_{j, j} j=1, \ldots, m$ & Setup cost $K_{j}, j=1, \ldots, m$ \\
\hline Demand assignment cost $d_{j i}$ & Transportation $\operatorname{cost} r_{j t}=d_{j i} \forall i, j, t$ \\
\hline & Production $\operatorname{cost} c_{j l}=0$ and $c_{j t}=m+1$ for $t=2, \ldots, n$ \\
\hline & Holding $\operatorname{cost} h_{j t}=0$, for $j=1, \ldots, m \quad t=1, \ldots, n$ \\
\hline & Demands $D_{t}=1$ \\
\hline
\end{tabular}

Now consider the solution of this specially constructed $\left(P^{\prime}{ }_{i}\right)$ instance. The following are true:

1. From Theorem 1 (the non-splitting property) we know that there exists an optimal solution to $\left(P^{\prime}{ }_{i}\right)$ where item $i^{\prime} s$ demand in period $t$ is produced in exactly one of the $J_{i}$ facilities. Since $c_{j l}=0$ and $c_{j t}=m+1$ for $t=2, \ldots, n$, production will only take place in period 1 at facility $j$. Demands in periods 2 to $n$ are satisfied completely by inventory carried over from period 1 , i.e., production in period $l$ at facility $j$ is $x_{j 1}=\sum_{t=2}^{n} D_{t} \delta_{j t}, \forall j$, and $I_{j t}=\sum_{\tau=t+1}^{n} D_{\tau} \delta_{j \tau}, \forall j, t$

2. Total transportation costs correspond to demand assignment costs i.e., $\sum_{t=1}^{n} \sum_{j=1}^{m} r_{j t} b_{j t}=\sum_{i=1}^{n} \sum_{j=1}^{m} d_{j i} y_{j i}$

3. Total setup cost corrspond to facility opening cost

It is easy to verify that the optimal value of the specially constructed $\left(P^{\prime}{ }_{i}\right)$ instance produces optimal value of the UFL instance. Thus, if the optimal solution of the $\left(P^{\prime}{ }_{i}\right)$ instance has a total cost less than $\kappa$, then there is a corresponding $U F L$ solution that has a total cost less then $\kappa$ (a yes answer to the decision problem). Otherwise, there is no assignment of demands to facilities with total cost lower than $\kappa$ (a no answer).

Since $U F L$ is known to be $N P$-complete, this proves that $\left(P_{i}^{\prime}\right)$ is also $N P$-complete. 